\title{
MICHAEL ATIYAH'S WORK IN ALGEBRAIC TOPOLOGY
}

\author{
GRAEME SEGAL
}

\begin{abstract}
In 1960 algebraic topology was at the centre of the mathematical stage, but Michael Atiyah burst into the field and changed its focus and its language. I describe his work of the following decade and its influence, keeping to the themes of $K$-theory and generalized cohomology to minimise the overlap with Dan Freed's account of Atiyah's work on index theory, which also appears in this issue.
\end{abstract}

In a number of talks and interviews (e.g., Min] Michael Atiyah spoke thoughtfully about his way of doing mathematics. He explained that he had rarely set out to solve a specific problem: rather he would be intrigued by some aspect of the mathematical landscape and feel driven to find out how it worked and get to the bottom of it. His work in algebraic topology exemplifies this very well. In the 1960 s, by inventing $K$-theory and the idea of a generalized cohomology theory, he changed the complexion of algebraic topology in the world, and, even after all his later work, I would say that the perspective of algebraic topology remained central in his mathematics. In his own eyes, however, he was a more general kind of geometer, who used the ideas of algebraic topology when they were needed. He felt the main stream of algebraic topology was too inward-looking, and held himself a little apart from it; but in the second half of his career he was always eager to apply his topologist's expertise in areas of mathematics which were new to him.

He began research in 1952 as an algebraic geometer. Though he was attracted by work in the older 'Italian' style, he wisely chose Hodge - the most 'modern' geometer in Cambridge - as his supervisor. Hodge had been deeply influenced by Lefschetz, who was at once the greatest exponent of topological ideas in algebraic geometry and the creator of much of modern algebraic topology. But Hodge's approach to topology was different from Lefschetz's. It focussed on the differential forms on algebraic varieties, and their integrals, combining de Rham's theorem with Chern's differential-geometric construction of characteristic classes. There are many parallels between Hodge's career and Michael's, and one of them is that Hodge's greatest triumph - the proof that every cohomology class (with real coefficients) of a closed smooth manifold has a unique harmonic form representative - is essentially a theorem of analysis, a field in which Hodge was not an expert and needed help from others.

Until 1959 Michael's work was in algebraic geometry, and though he expressed it in the modern language of topology, treating ruled surfaces as fibre bundles and speaking of 'vector bundles' rather than 'linear systems', his methods were algebraic. His thesis, submitted in 1955, on algebraic differential forms, displayed a command of spectral sequences and of the cohomology theory of sheaves.

Received by the editors June 10, 2021.

2020 Mathematics Subject Classification. Primary 55-XX, 58-XX. 
One paper from this period stands out for illustrating so well his knack, throughout his career, of being the first to put his finger on an essential point. In [AtCl] he shows that the characteristic classes of a holomorphic vector bundle $E$ on a complex manifold $X$ all come from one basic class $\operatorname{At}(E) \in H^{1}\left(X ; \Omega^{1}(\operatorname{End}(E))\right)$-now called the Atiyah class - which describes the extension of sheaves (or of holomorphic vector bundles 1 on $X$

$$
\Omega^{1}(E) \rightarrow J^{1}(E) \rightarrow E .
$$

The class $\operatorname{At}(E)$ is the holomorphic analogue of the curvature $K_{E} \in \Omega^{2}(\operatorname{End}(E))$ defined in the differentiable context for a bundle with a connection. The characteristic classes of $E$, in the Dolbeault cohomology $H^{*}\left(X ; \Omega^{*}\right)$, are obtained from $\operatorname{At}(E)$ as $P(\operatorname{At}(E)) \in H^{p}\left(X ; \Omega^{p}\right)$, where $P: \operatorname{End}\left(\mathbb{C}^{n}\right) \rightarrow \mathbb{C}$ is a polynomial of degree $p$ invariant under conjugation by $G L_{n}(\mathbb{C})$, just as the Chern-Weil description of the corresponding classes in de Rham cohomology is $P\left(\frac{1}{2 \pi} K_{E}\right) \in H^{2 p}(X ; \mathbb{R})$.

The best-known of Michael's early papers, however, is his study EllCve of the moduli space of holomorphic bundles on an elliptic curve, which foreshadows his later interest in gauge theory. It is described in Simon Donaldson's contribution to this volume [Do].

Immediately after finishing his thesis, Michael spent the year 1955-56 at the Institute for Advanced Study (IAS) in Princeton. He often wrote of the enormous stimulation he got from meeting the young stars Serre, Milnor, Hirzebruch, Bott, and Singer, as well as the older mathematicians Kodaira and Spencer. The 1950s were a decade of spectacular flowering of algebraic topology, and for anyone interested in geometry it must have seemed the most exciting thing on the mathematical stage. Among the main developments - all being worked on in Princeton, and all to prove relevant to Michael's work - were

- the reformulation of the subject in the language of category theory;

- the homotopy-theoretic understanding of fibre bundles and their classifying spaces and characteristic classes;

- Thom's invention of the cobordism ring, and the reduction of its calculation to homotopy theory;

- the use of cohomology operations, and especially the Steenrod algebra, to calculate the cobordism ring, the development of the Adams spectral sequence, and the solution of the Hopf invariant problem;

- Bott's application of Morse theory to the topology of Lie groups, culminating in his periodicity theorem;

- Milnor's discovery of exotic smooth structures on spheres, and his work with Kervaire relating differential topology to the homotopy theory of spheres and Grassmannians.

\section{The Beginnings of $K$-THEORY}

Of the mathematicians Michael met in Princeton, Hirzebruch (who had corresponded with Todd and given the Todd classes their name) was the nearest to him in mathematical background and became his close collaborator for the next few years.

\footnotetext{
${ }^{1}$ Here the bundle $E$ is identified with its sheaf of holomorphic sections, while $\Omega^{1}(E)$ is the sheaf of holomorphic 1-forms with values in $E$, and $J^{1}(E)$ is the sheaf of 1-jets of holomorphic sections of $E$. We observe that the sheaf of homomorphisms from $E$ to $\Omega^{1}(E)$, whose first cohomology is the home of the extension-class, can be identified with $\Omega^{1}(\operatorname{End}(E))$
} 
A little older than Michael, he was already a considerable figure in the mathematical world. He was an expert in algebraic topology, as well as in sheaf-theoretic methods in complex analysis. He had developed a very practical calculus for encoding the multiplicative characteristic classes of vector bundles as formal power series, which enabled him to read off a formula for the signature of a compact oriented smooth manifold - or indeed for any multiplicative cobordism invariant - in terms of the Pontryagin classes of the tangent bundle. But he was most famous for his 'higher-dimensional Riemann-Roch' theorem, which, for a holomorphic vector bundle $E$ on a compact complex manifold $M$, expresses the Euler number

$$
\chi(M ; E)=\sum(-1)^{q} \operatorname{dim} H^{q}(M ; E)
$$

in terms of the Chern classes of $E$ and of the tangent bundle $T_{M}$ of $M$. (Here $H^{q}(M ; E)$ is the cohomology of $M$ with coefficients in the sheaf of holomorphic sections of $E$.)

Hirzebruch's calculus of characteristic classes. As Hirzebruch's calculus of characteristic classes is so central in Michael's work, I shall interpolate here a brief account of it. A characteristic class $\Phi$ for complex vector bundles, with coefficients in a commutative ring $A$, assigns to a bundle $E$ on a space $X$ a cohomology class $\Phi(E) \in H^{*}(X ; A)$. Because an $n$-dimensional bundle $E$ can be described by a map $f_{E}$ - unique up to homotopy - from $X$ to the classifying space or Grassmannian $B U_{n}$, we can describe $\Phi$ by a sequence of classes $\Phi_{n} \in H^{*}\left(B U_{n} ; A\right)$ such that $\Phi(E)=f_{E}^{*} \Phi_{n}$. The characteristic class is called additive if $\Phi(E \oplus F)=\Phi(E)+\Phi(F)$ and multiplicative if $\Phi(E \oplus F)=\Phi(E) . \Phi(F)$.

Because the direct-sum map

$$
B U_{1} \times \cdots \times B U_{1} \rightarrow B U_{n}
$$

induces an injection in cohomology, any characteristic class is determined by its values on sums of line bundles, and a multiplicative class $\Phi$ is determined by the single power-series $\Phi_{1} \in H^{*}\left(B U_{1} ; A\right)=A[[t]]$. In fact $H^{*}\left(B U_{n} ; A\right)$ is just the subring of elements in

$$
H^{*}\left(B U_{1} \times \cdots \times B U_{1} ; A\right)=A\left[\left[t_{1}, \ldots, t_{n}\right]\right],
$$

which are symmetric under permuting $t_{1}, \ldots, t_{n}$, so any choice of $\Phi_{1}$ gives rise to the multiplicative class with $\Phi_{n}=\prod_{i} \Phi_{1}\left(t_{i}\right)$, and we can rewrite this as a function of the elementary symmetric functions $c_{1}, \ldots, c_{n}$ of $t_{1}, \ldots, t_{n}$, which are the Chern classes of the bundle.

A multiplicative class $\Phi$ is stable, i.e., $\Phi(E)$ does not change if a trivial bundle is added to $E$, if and only if the power-series $\Phi_{1}$ has constant term 1 . In this case we can think of $\Phi$ as an element of the cohomology of the stabilized classifying space $B U=\bigcup_{n} B U_{n}$, and $\Phi$ defines a ring-homomorphism $H_{*}(B U ; \mathbb{Z}) \rightarrow A$.

The picture for multiplicative characteristic classes of real vector bundles is little different, as long as the bundles are orientable and we use a coefficient $\operatorname{ring} A$ in which 2 is invertible. We must replace $B U_{n}$ by $B S O_{n}$, but the upshot is just that stable multiplicative classes correspond to the even power-series $A\left[\left[t^{2}\right]\right] \subset A[[t]]$.

Finally, multiplicative characteristic classes in rational cohomology are essentially the same thing as rational-valued genera $\Theta$, i.e., ring-homomorphisms

$$
\Theta: \Omega_{*}^{S O} \rightarrow \mathbb{Q}
$$


from Thom's ring $\Omega_{*}^{S O}$ of cobordism classes of oriented manifolds. For there is a ring-homomorphism

$$
\Omega_{*}^{S O} \rightarrow H_{*}(B S O ; \mathbb{Z})
$$

which associates to a closed oriented $n$-manifold $M$ the image of its fundamental homology class $[M] \in H_{n}(M)$ under the classifying map $M \rightarrow B S O_{n} \rightarrow B S O$ of its tangent bundle, and Thom proved that this map becomes an isomorphism when tensored with $\mathbb{Q}$.

The multiplicative characteristic classes that will come into this account are

- the total Chern class $1+c_{1}+c_{2}+\cdots$, corresponding to the series $1+t$;

- Hirzebruch's L-class for real bundles, corresponding to the series $t \operatorname{coth} t$, which gives his formula for the signature;

- the Todd class, corresponding to the series $t /\left(1-\mathrm{e}^{-t}\right)$;

- the $\hat{A}$-class for real bundles, corresponding to the series $(t / 2) / \sinh (t / 2)$;

- the Euler class of an oriented even-dimensional real bundle, which is multiplicative but not stable - it vanishes if the bundle has a nonvanishing section - and corresponds to the element $t \in \mathrm{H}^{2}\left(\mathrm{BSO}_{2} ; \mathbb{Z}\right)$.

Apart from these multiplicative classes, a central role is played by the Chern character ch, given by the symmetric function

$$
\sum_{i} \mathrm{e}^{t_{i}} \in H^{*}\left(B U_{1} \times \cdots \times B U_{1} ; \mathbb{Q}\right) .
$$

It has the properties

$$
\begin{gathered}
\operatorname{ch}(E \oplus F)=\operatorname{ch}(E)+\operatorname{ch}(F), \\
\operatorname{ch}(E \otimes F)=\operatorname{ch}(E) \cdot \operatorname{ch}(F) .
\end{gathered}
$$

Returning to our story, we can now state the Riemann-Roch formula:

$$
\chi(M ; E)=\left\langle\operatorname{ch}(E) \operatorname{Todd}\left(T_{M}\right),[M]\right\rangle .
$$

Hirzebruch saw that the formula implies that the Euler number $\chi(M ; E)$, which prima facie depends on the very rigid holomorphic structure of $M$ and $E$, is actually a purely topological invariant of the topological space $M$ and the two complex vector bundles $E$ and $T_{M}$. Because $\chi(M ; E)$ is an integer, while the RiemannRoch expression for it is a polynomial with rational coefficients in the Chern classes, the theorem implies a rich array of congruences between the topologically defined characteristic numbers of the manifold. These look like strong constraints on its topology. It was natural to ask whether they hold for all smooth manifolds, or at least for a larger class than just smooth algebraic varieties.

When their active collaboration began, Atiyah and Hirzebruch were both thinking about two of the important developments of 1957-58: Grothendieck's generalization of Hirzebruch's theorem, and Bott's periodicity theorem, which gave an explicit homotopy equivalence

$$
\mathbb{Z} \times B U \rightarrow \Omega^{2}(\mathbb{Z} \times B U)
$$

between the stabilized classifying space $\mathbb{Z} \times B U$ of the unitary groups and its twofold loop-space. The two results were not obviously related. 
Grothendieck's theorem concerned a proper map $f: X \rightarrow Y$ of smooth algebraic varieties, and it was stated in terms of Grothendieck groups. For any category $\mathcal{C}$ in which there is a notion of short exact sequence,

$$
0 \rightarrow E^{\prime} \rightarrow E \rightarrow E^{\prime \prime} \rightarrow 0,
$$

the Grothendieck group of $\mathcal{C}$ is the abelian group generated by the isomorphism classes $[E]$ of objects of $\mathcal{C}$, subject to the relations $[E]=\left[E^{\prime}\right]+\left[E^{\prime \prime}\right]$ for every exact sequence. Grothendieck applied this construction to the category of coherent sheaves on a variety $X$ to define his $K$-group $K_{\text {alg }}(X)$, and observed that for a smooth variety over the complex numbers it is related to the cohomology of $X$ as a topological space by the Chern character map

$$
\operatorname{ch}: K_{\mathrm{alg}}(X) \rightarrow \bigoplus_{k} H^{2 k}(X ; \mathbb{Q}) .
$$

Coherent sheaves can be pushed forward by proper maps, and Grothendieck showed that a proper map $f: X \rightarrow Y$ induces a homomorphism 2

$$
f_{!}: K_{\text {alg }}(X) \rightarrow K_{\text {alg }}(Y)
$$

closely related to the Gysin homomorphism $f_{*}: H^{*}(X ; \mathbb{Q}) \rightarrow H^{*}(Y ; \mathbb{Q})$, which is the map induced by $f$ on homology regarded as a map of cohomology by using the Poincaré duality isomorphisms of both $X$ and $Y$. Grothendieck's theorem asserts that the diagram

$$
\begin{array}{rlc}
K_{\mathrm{alg}}(X) & \stackrel{f_{!}}{\longrightarrow} & K_{\mathrm{alg}}(Y) \\
\mathrm{ch} \downarrow & & \operatorname{ch} \downarrow \\
H^{*}(X ; \mathbb{Q}) & \stackrel{f_{*}}{\longrightarrow} & H^{*}(Y ; \mathbb{Q})
\end{array}
$$

does not quite commute, but commutes if the Chern character maps on the left and right are multiplied by the invertible elements $\operatorname{Todd}\left(T_{X}\right)$ and $\operatorname{Todd}\left(T_{Y}\right)$ of the respective cohomology rings. Hirzebruch's Riemann-Roch theorem is contained in Grothendieck's as the case when $Y$ is a point, for a coherent sheaf on a point is simply a finite-dimensional vector space, whose Chern character is its dimension, and $f_{!}(E)$ is the alternating sum of the cohomology groups $H^{i}(X ; E)$.

The strategy of Grothendieck's proof was to have a lasting influence on Michael's work. If the theorem holds for maps $f: X \rightarrow Y$ and $g: Y \rightarrow Z$, then it obviously holds for $g \circ f$. But a projective variety $X$ has an embedding $i: X \rightarrow \mathbb{P}^{m}$ in projective space, so $f: X \rightarrow Y$ can be factorized

$$
X \rightarrow Y \times \mathbb{P}^{m} \rightarrow Y,
$$

where the first map is $f \times i$ and the second is the projection. It is enough, therefore, to prove the theorem for an embedding and for the projection of a product with $\mathbb{P}^{m}$. In the differentiable category, because a closed manifold can be embedded in a sphere, the analogous factorization of a map $f: X \rightarrow Y$ of smooth manifolds is $X \rightarrow Y \times S^{2 m} \rightarrow Y$, and Michael had the idea that the natural way to push vector bundles forward under the projection $Y \times S^{2 m} \rightarrow Y$ is by Bott periodicity. This was a very new angle on Bott's theorem, which in the topological world was mainly seen in the light of Milnor's immediate recognition [BoM] of it as the crucial fact of

\footnotetext{
${ }^{2}$ It is not, however, simply the push-forward $f_{*}$ of sheaves which induces Grothendieck's map $f_{\text {! }}$ on $K_{\text {alg. }}$. Because $f_{*}$ is not an exact functor, $f$ ! must be defined as the alternating sum of the right-derived functors of $f_{*}$.
} 
homotopy theory needed to prove the nonparallelizability of spheres of dimension greater than 7 .

Atiyah and Hirzebruch put Grothendieck's theorem together with Bott periodicity and obtained a differentiable Riemann-Roch theorem 3 DiffRR which answered many of the questions about the integrality of characteristic numbers mentioned above. It also put in the foreground the relevance of a spin structure on the manifold - a 'higher' kind of orientability - for integrality properties, and (using the version for real vector bundles which was part of the announcement) it gave a new proof of Rokhlin's theorem that the signature of a 4-manifold is divisible by 16 if it has a spin structure. Nevertheless, in its first incarnation the differentiable theorem was not very geometrical or illuminating. It conjectured-and, sufficiently for most applications, established - that if a map $f: X \rightarrow Y$ of differentiable manifolds satisfies a certain spin-orientability condition, one can associate to it a map $f_{!}: K(X) \rightarrow K(Y)$ of Grothendieck groups of vector bundles which can be calculated cohomologically by the same formula which Grothendieck found in the algebraic case. But the announcement gave no reason for the existence of $f_{!}$- indeed it asserted discouragingly that in the differentiable case there is no analogue of the holomorphic (or algebraic) operation of pushing forward a coherent sheaf. This pessimism perhaps came from defining $f_{\text {! differently for embeddings }}$ and projections: it would have been more accurate to say that in the differentiable context there was no analogue of coherent sheaves. Understanding the situation better was to be the goal of much of Michael's work for more than ten years, and it led him into index theory, which, as he later remarked, is "really the same thing as $K$-theory".

In formulating their theorem, Atiyah and Hirzebruch invented the ' $K$-theory' of algebraic topology, and with it the idea of a generalized cohomology theory. Both ideas immediately took hold in algebraic topology, and it is worth reflecting on the reasons. After Grothendieck's work it cannot have been such a step to consider the Grothendieck group $K(X)$ of vector bundles on a compact space $X$, and it was known that this could be identified with $[X ; \mathbb{Z} \times B U]$, the homotopy classes of maps from $X$ to $\mathbb{Z} \times B U$. Puppe had recently published his semi-infinite exact sequence $\mathrm{P}$ for any such functor, and Bott's theorem immediately extended this to the doubly infinite sequence which is the defining property of a generalized cohomology theory. Furthermore, Barratt [B] and others had —-without using the words - already shown that the stable homotopy classes of maps from a varying compact space into any given fixed space do indeed form a generalized cohomology theory. So in some sense the crucial point was a matter of language: the new concepts were ideally suited to formulate the ideas then at the forefront of algebraic topology. It certainly helped that both Atiyah and Hirzebruch were superb and charismatic lecturers.

That, though, is the perspective of hindsight. In his own commentaries Michael wrote that "introducing the odd-dimensional $K$-groups seemed at the time a daring generalization". We should remember, too, that nothing about Grothendieck's group $K(X)$ suggested it could be just one component of a $\mathbb{Z}$-graded group: its elements already included the classes of algebraic cycles of all dimensions in $X$. Indeed the splitting of $K(X)$ into its cohomological components as eigenspaces of the Adams operations, with the topological dimension arising as an eigenvalue,

\footnotetext{
${ }^{3}$ The first announcement was Hirzebruch's Bourbaki seminar in February 1959.
} 
seems to me one of the enduring mysteries of the subject. We shall return to this in $\S 3$ and $\S 7$.

Of course the new theory would not have had such impact without the applications that came with it. Probably the most striking early application was made by Frank Adams, Michael's rival from undergraduate days at Trinity College, Cambridge, who in 1961 used $K$-theory to prove the long-conjectured theorem that the maximum number of linearly independent tangent vector fields on a sphere $S^{n-1}$ is the Radon-Hurwitz number $c_{n}$. (Clifford algebras and spinors appeared here once again in connection with $K$-theory, for $c_{n}$ is the largest number $k$ such that the Clifford algebra with $k$ generator 4 acts on the vector space $\mathbb{R}^{n}$.) Adams, with the invention of the Adams spectral sequence and the solution of the Hopf invariant problem in 1958, was already established as a leader in algebraic topology, but with hindsight it seems fair to say that the vector fields theorem was a fruit ready to be plucked in 1961. The question is to determine for which $k$ there is a cross-section of the forgetful map

$$
S O_{n} / S O_{n-k-1} \rightarrow S O_{n} / S O_{n-1}=S^{n-1}
$$

from the Stiefel manifold of orthonormal $(k+1)$-tuples of vectors in $\mathbb{R}^{n}$ to the unit sphere. Ioan James [J] had made a crucial reduction of the problem to one about the "stunted projective spaces" $\mathbb{P}^{n} / \mathbb{P}^{n-k-1}$, and Michael had reworked this in ThCpl into a convenient language of Thom complexes, and in his paper At-Todd with Todd had solved the analogous but simpler problem for the unitary groups.

Michael's paper ThCpl on Thom complexes is perhaps the one where he writes most like an orthodox algebraic topologist. It is worth dwelling on because, without proving any deep new theorem, it was influential in steering the evolution of the subject towards the Atiyah perspective. The Thom space $X^{E}$ of a real vector bundle $E$ on a compact space $X$ is the one-point compactification of the space $E$, and ThCpl develops the idea of $X^{E}$ as a twisted suspension of $X$. (If $E$ is the trivial bundle $X \times \mathbb{R}^{m}$, then $X^{E}$ is the $m$-fold suspension of $X$.) It goes on to show that in the stable homotopy category $X^{E}$ is well-defined even when $E$ is replaced by a virtual bundle, i.e., the formal difference $E_{1}-E_{2}$ of two vector bundles. Most importantly, it shows that in the stable homotopy category (which is an additive category), the dual object to a closed $n$-manifold $X$ is the Thom space $X^{-T_{X}}$, where $T_{X}$ is the tangent bundle of $X$. If $X$ is embedded in $\mathbb{R}^{n+k}$, the dual of $X$ is therefore the $(n+k)$-fold desuspension of the Thom space $X^{N_{X}}$ of the normal bundle $N_{X}$. Thus $H_{n-i}(X) \cong H^{k+i}\left(X^{N_{X}}\right)$, and if $X$ is orientable, the fundamental class $[X] \in H_{n}(X)$ corresponds to the Thom class $\theta_{N_{X}} \in H^{k}\left(X^{N_{X}}\right)$.

The Thom space $X^{E}$ of a real vector bundle $E$ can also be described as the mapping cone of $E^{\circ} \rightarrow X$, where $E^{\circ}$ is the complement of the zero-section in $E$, and is a spherical fibration on $X$, i.e., a bundle whose fibres are homotopy-spheres. Thus a Thom space can be defined for any spherical fibration, and its stable homotopy type depends only on the stable fibre-homotopy type of the spherical fibration. Michael pointed out the invariance of $X^{E}$ under fibre-homotopy equivalences of $E^{\circ}$ (I shall return to this when discussing operations in $K$-theory below) but stopped short of discovering the Spivak normal fibration 5 When a compact space $X$ is embedded in $\mathbb{R}^{m}$ as a neighbourhood deformation retract of an open subset $U_{X}$,

\footnotetext{
${ }^{4}$ This means that we have anticommuting skew $n \times n$ matrices $e_{1}, \ldots, e_{k}$ such that $e_{1}^{2}=\cdots=$ $e_{k}^{2}=-1$. If $\xi \in \mathbb{R}^{n}$ is a point of $S^{n-1}$ this gives us $k$ tangent vectors $e_{i} \xi$ at $\xi$.

${ }^{5}$ This is described in Spivak's 1964 thesis, and is attributed there to his advisor Milnor.
} 
the dual of $X$ in the stable homotopy category is the one-point compactification $U_{X}^{+}$, and the complement $U_{X} \backslash X$ is a spherical fibration-the Spivak fibration of $X$-if and only if $X$ satisfies Poincaré duality. (The proof of this striking example of a global condition implying a property that looks local is almost obvious if $X$ is simply-connected, but depends on defining carefully what is meant by Poincaré duality when there is a fundamental group.)

Generalized cohomology theories are not mentioned in [ThCpl, though it was submitted only two weeks before his paper BordCob on bordism theories. Looking at ThCpl now, I wondered why it fails to point out that it effectively contains a simple proof of the differentiable Riemann-Roch theorem, which was left in a somewhat unsatisfactory state in the preceding papers [DiffRR] and [Tucs. I shall give the argument and then speculate about Michael's lack of enthusiasm for it.

An $h^{*}$-orientation of a rank $k$ real vector bundle $\pi: E \rightarrow X$, for any multiplicative cohomology theory $h^{*}$, is a choice of a Thom class $\theta_{E} \in h^{k}\left(X^{E}, \infty\right)=h_{\mathrm{cpt}}^{k}(E)$, i.e., a class which restricts to a generator of $h_{\mathrm{cpt}}^{k}\left(E_{x}\right) \cong h^{0}$ (point) for each fibre of $E$. (Here I have used the idea of cohomology with compact supports, which, for a locally compact space $Y$, is defined by $h_{\mathrm{cpt}}^{*}(Y)=h^{*}\left(Y^{+}, \infty\right)$.) The reason for the terminology is that the two generators of $H_{\mathrm{cpt}}^{k}\left(\mathbb{R}^{k} ; \mathbb{Z}\right) \cong \mathbb{Z}$ correspond to the orientations of $\mathbb{R}^{k}$. Because $h_{\mathrm{cpt}}^{*}(Y)$ is a module over the ring $h^{*}(Y)$, a Thom class $\theta_{E}$ defines an isomorphism $\xi \mapsto \pi^{*}(\xi) . \theta_{E}$ from $h^{*}(X)$ to $h_{\mathrm{cpt}}^{*+k}(E)$.

When we have a map of closed manifolds $f: X \rightarrow Y$, we can embed $Y$ in some $\mathbb{R}^{m}$, and then, if $m$ is large compared with the dimension of $X$, an arbitrarily small deformation of $f$ will be an embedding of $X$ - and hence of its tubular neighbourhood $N_{X}$-inside the tubular neighbourhood $N_{Y} \subset \mathbb{R}^{m}$ of $Y$. Because one-point compactification is a contravariant functor for open embeddings, this means that $f$ induces a canonical map

$$
\check{f}: Y^{N_{Y}} \rightarrow X^{N_{X}}
$$

in the stable homotopy category. If $N_{X}$ and $N_{Y}$ are $h^{*}$-oriented, then combining $\check{f}$ with the Thom isomorphisms gives us the desired map

$$
f_{!}: h^{*}(X) \rightarrow h^{*}(Y) .
$$

In fact, if the Thom classes $\theta_{E}$ are multiplicative for direct sums of bundles, as is usually the case, then we need only the virtual bundle $T_{X}-T_{Y}=N_{Y}-N_{X}$ to be $h^{*}$-oriented.

Finally, if we have two multiplicative theories $k^{*}$ and $h^{*}$ and a multiplicative transformation $\Psi: k^{*} \rightarrow h^{*}$, then we get a multiplicative characteristic class $E \mapsto$ $\hat{\Psi}(E)$ with values in $h^{*}$, defined on the class of bundles oriented for both theories, by 6

$$
\Psi\left(\theta_{E}^{(k)}\right)=\hat{\Psi}(-E) \cdot \theta_{E}^{(h)} .
$$

This gives us an abstract Riemann-Roch theorem (which Atiyah and Hirzebruch were to apply to multiplicative automorphisms of classical cohomology in CohOps,

\footnotetext{
${ }^{6}$ The reason for the minus sign before $E$ is that we want $\hat{\Psi}\left(T_{X}\right)$ to be associated to the Thom isomorphism for the normal bundle of $X$.
} 
cf. $\S 7$ below) asserting that the diagram

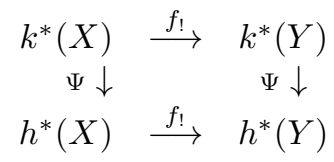

commutes when the vertical maps are multiplied by $\hat{\Psi}\left(T_{X}\right)$ and $\hat{\Psi}\left(T_{Y}\right)$.

The extensive work BorH] on characteristic classes by Borel and Hirzebruch had implicitly established that a real vector bundle $E$ is orientable for the theory $K^{*}$ only when it has a spin ${ }^{\mathbb{C}}$-structure, or, equivalently, when the Stiefel-Whitney class $w_{2}(E)$ lifts to an integral class. Indeed their work associates a specific Thom class $\theta_{E}^{(K)}$ to a spin ${ }^{\mathbb{C}}$-structure, and it has the property that

$$
\operatorname{ch} \theta_{E}^{(K)}=\hat{A}(-E) \cdot \theta_{E}^{(H)},
$$

where $\theta_{E}^{(H)}$ is the unique classical Thom class defined by the orientation of $E$. So the abstract theorem gives a complete proof of the differentiable RiemannRoch theorem, adequate for all the applications that had been made of it, e.g., in Michael's paper ImmEmb] on immersions and embedding of manifolds.

Michael would not have liked the way this account treated $K$-theory as just one among a class of cohomology theories. He saw $K$-theory as a very special theory with a deep basis in analysis related to Bott periodicity. He was convinced that it was simpler and more natural than classical cohomology. A $K$-theory class, he felt, represents a natural geometric object - a vector bundle - whereas a classical cohomology class involves an elaborate algebraic structure. He wanted to see the $K$-theory Gysin maps, too, as natural geometric operations, which would imply, rather than follow from, the cohomological calculations of [BorH].

He developed these ideas in a series of papers in the 1960s, and in his pedagogical book [KTh, which completely avoids using classical cohomology. By nature he was impatient of foundational material, and these works present not so much foundations as a point of view. I shall review a number of its strands in turn:

(§2) calculating the $K$-theory of geometrically important spaces and showing how it reflects and illuminates the geometry;

$(\S 3-\S 5)$ elaborating the nature of $K$-theory classes and their relation to the dual $K$-homology classes, and especially the relation of the Gysin map to Bott periodicity;

(§6) equivariant $K$-theory;

$(\S 7)$ the natural operations in $K$-theory;

(§8) the $K$-theory of real vector bundles and the role of Clifford algebras.

\section{Calculations}

The first systematic account Tucs of $K$-theory was written in early 1960 . It defines $K(X)=K^{0}(X)$ for a finite CW-complex $X$ as the Grothendieck group of vector bundles on $X$, and points out that the tensor product of bundles makes $K(X)$ a commutative ring. Then $K^{-i}(X)$ is defined for $i>0$ as the (reduced) $K^{0}$ of the $i$-fold suspension of $X$. The Bott periodicity theorem can be stated as $K^{-i}(X) \cong K^{-i-2}(X)$, and this is used to define the groups for all $i \in \mathbb{Z}$. The axioms of a cohomology theory are verified by using the Puppe sequence already mentioned. The paper continues by establishing the Atiyah-Hirzebruch spectral 
sequence, which shows how close $K$-theory is to classical integral cohomology. In particular, for a compact space $X$ the Chern character of a vector bundle induces an isomorphism

$$
K^{*}(X) \otimes \mathbb{Q} \rightarrow H^{*}(X ; \mathbb{Q}) .
$$

Thus, as an additive group, $K^{*}(X)$ differs from $H^{*}(X ; \mathbb{Z})$ only by torsion. The spectral sequence is related to the natural decreasing filtration

$$
K^{*}(X) \supset K_{(1)}^{*}(X) \supset K_{(2)}^{*}(X) \supset \cdots,
$$

where $K_{(p)}^{*}(X)$ consists of the elements which vanish when pulled back to any space of dimension less than $p$. If, for example, the cohomology has no torsion, or if $H^{i}(X ; \mathbb{Z})$ vanishes when $i$ is odd, the spectral sequence shows that the associated graded group of the natural filtration of $K^{i}(X)$ is

$$
\bigoplus_{k \in \mathbb{Z}} H^{i+2 k}(X ; \mathbb{Z})
$$

The next topic in [Tucs] is the Gysin map $f_{!}: K^{*}(X) \rightarrow K^{*}(Y)$ induced by a map $f: X \rightarrow Y$ of compact smooth manifolds which satisfies a spin-orientability condition. The treatment of this essential ingredient in the differentiable Riemann-Roch theorem of [DiffRR], however, is still confessedly provisional and unsatisfactory, and was soon to be superseded by the viewpoint of ThCpl discussed above.

After this foundational material the paper turns to its main objective, the calculation of the $K$-theory of some geometrically interesting spaces. The foundational part is written very much in the language of the homotopy theory of the time, and is less directly geometrical than Michael would have made it a few years later: it does not mention that every vector bundle $E$ on a compact space has a complementary bundle $F$ such that $E \oplus F$ is trivial, and it does not use the difference-bundle description of a relative class in $K(X, Y)$, which I shall discuss in the next section.

The first space considered in Tucs, is the classifying space $B G$ of a compact connected Lie group $G$. The cohomology of $B G$ had been studied throughout the 1950 s, most intensively by Borel and Hirzebruch BorH. The results were complicated: even for $B S O_{n}$ it is not so easy to describe the integral cohomology ring explicitly, and the result for $B \operatorname{Spin}_{n}$ was unknown until Quillen's work Q1. For $K$-theory the situation is very different. Every finite-dimensional complex representation $V$ of $G$ defines a vector bundle $E_{V}$ on $B G$, and the assignment $V \mapsto E_{V}$ induces a ring-homomorphism $R(G) \rightarrow K^{0}(B G)$ from the representation-ring $R(G)$, the Grothendieck group of the category of finite-dimensional representations, i.e., the free abelian group generated by the classes of irreducible representations. It turns out that $R(G) \rightarrow K^{0}(B G)$ is close to being an isomorphism: this is easy to prove when $G$ is connected, because if $T$ is a maximal torus in $G$, then $R(G)$ and $K^{0}(B G)$ restrict injectively into the explicitly known groups $R(T)$ and $K^{0}(B T)$, the image in each case being the subgroup invariant under the conjugation-action of the Weyl group of $G$ on $T$. The only problem is that the space $B G$ is not compact, and so one cannot expect every element of its $K$-theory to be represented by the difference between two finite-dimensional vector bundles. For a noncompact space $X$ the correct definition of $K^{0}(X)$ is as $[X ; \mathbb{Z} \times B U]$, but when $X=B G$ this is equivalent to another plausible definition as the inverse-limit of a system $\left\{K^{0}\left(B_{n} G\right)\right\}$, where $\left\{B_{n} G\right\}$ is any expanding family of compact subspaces whose union is $B G$. The paper uses the inverse-limit definition, which makes it obvious 
that $K^{0}(B G)$ is complete in the topology induced by its natural filtration, and this motivates the theorem that

$$
R(G)^{\wedge} \cong K^{0}(B G)
$$

where $R(G)^{\wedge}$ is the completion of the representation ring in a topology which we shall return to in $\S 7$. The other $K$-group $K^{1}(B G)$ vanishes.

From $B G$ the paper turns to the homogeneous spaces $G / H$, where $G$ is a connected and simply-connected compact Lie group and $H$ is a subgroup of maximal rank, i.e., one which contains a maximal torus $T$ of $G$. This is the class of spaces typified by the projective spaces, Grassmannians, and flag manifolds long familiar in algebraic geometry. They are complex projective algebraic varieties with natural decompositions into Schubert cells, each of which is a complex affine space. Thus their integral cohomology is all in even dimensions, and is a free abelian group with a canonical basis.

Every finite-dimensional complex representation $V$ of $H$ defines a holomorphic vector bundle $E_{V}=G \times_{H} V$ on $G / H$, and the bundles so arising are called homogeneous. This gives us a ring-homomorphism

$$
R(H) \rightarrow K^{0}(G / H),
$$

which corresponds to the map induced in $K$-theory by the classifying map $G / H \rightarrow$ $B H$ of the principal $H$-bundle $G \rightarrow G / H$. If the action of $H$ on $V$ extends to a representation of $G$, then the bundle $E_{V}=G \times{ }_{H} V \cong(G / H) \times V$ is a trivial bundle of the dimension of $V$. So the preceding ring-homomorphism factorizes

$$
R(H) \rightarrow R(H) \otimes_{R(G)} \mathbb{Z} \rightarrow K^{0}(G / H),
$$

where $R(G)$ acts on $R(H)$ by restriction, and on $\mathbb{Z}$ by the augmentation-homomorphism which takes a representation to its dimension. The paper conjectures, correctly, that the second map is an isomorphism, and proves it in many cases. Once again, nothing remotely so simple is true for the classical cohomology rings of the spaces $G / H$. (This theorem had a back-influence in algebra too, leading Steinberg to his elegant proof $[\underline{\mathrm{S}}$ that $R(H)$ is a free module over $R(G)$ with an explicit basis indexed by the Schubert cells in $G / H$ : when $H$ is the maximal torus $T$, for instance, the basis element corresponding to a cell $C$ is just the determinant of the representation of $T$ on the tangent space to $C$.)

After calculating the $K$-theory of $B G$ for a connected compact group $G$, it was natural to ask about other groups, and Michael proved the same result $R(G)^{\wedge} \cong$ $K^{0}(B G)$ for all finite groups $G$ in his paper [FiniteG, which was written almost simultaneously with Tucs. The finite-group case was much harder to prove, and used quite different ideas. Michael sought for a more illuminating proof, and a few years later this was found with the invention of equivariant $K$-theory described in $\S 6$. One new feature of the finite group case especially attracted his attention: for a finite group the completion $R(G) \rightarrow R(G)^{\wedge}$ loses information. An element of $R(G)$ is determined by its character, and indeed $R(G) \otimes \mathbb{C}$ can be identified with the conjugation-invariant complex-valued functions on $G$; but in the completion all that is retained is the values of the characters on the elements of prime-power order in $G$. In more modern language: the space $B G$ breaks up as a product $\prod_{p} B_{(p)} G$ over the primes $p$, where $B_{(p)} G$ is a space constructed entirely from the lattice of $p$-subgroups of $G$. This means that the classifying space $B G$ does not see all the subtlety of the interaction between elements of $G$ whose orders are powers of 
different primes. The hope of doing better than this, and of being able to prove significant theorems about finite groups by $K$-theory, was one of the incentives in developing equivariant $K$-theory.

We have been considering the $K$-theory of spaces related to a compact Lie group $G$, but so far not $K^{*}(G)$ itself. This too has a simple description, as least when $G$ is connected and simply-connected. A finite-dimensional complex representation defines not only a vector bundle on $B G$ but also its transgression, an element of $K^{-1}(G)$, for the suspension of $G$ is naturally a subspace of $B G$. (Or, more simply, a homomorphism $G \rightarrow U_{n}$ gives a way of attaching, base-to-base, two copies of the trivial bundle $C G \times \mathbb{C}^{n}$ on the cone $C G$ on $G$ to form a bundle on the suspension of $G)$. When $G$ is simply-connected, $R(G)$ is the polynomial ring generated by $r$ 'basic' irreducible representations $\rho_{1}, \ldots, \rho_{r}$, where $r$ is the rank of $G$, and identifying the $\rho_{i}$ with classes in $K^{-1}(G)$ gives us a ring-homomorphism

$$
\bigwedge_{\mathbb{Z}}\left(\rho_{1}, \ldots, \rho_{r}\right) \rightarrow K^{*}(G) .
$$

In 1962 Michael's student Luke Hodgkin [Ho proved that this map is an isomorphism. The proof was complicated, using the classification of Lie groups, the difficult part being to prove that $K^{*}(G)$ has no torsion. In 1965 Michael published an ingenious and elegant short proof that the map (1) is an isomorphism onto $K^{*}(G) /$ (torsion). For a long time no easy way was found to rule out the existence of torsion, but, more than fifty years later, at the age of 85 , Michael eagerly told me of a paper of Baraglia and Hekmati $\mathrm{BarH}$ ] which gave a completely new proof of the theorem by observing that if $G$ is regarded as a torus bundle over $G / T$, then the fibrewise Fourier-Mukai transformation maps $K^{*}(G)$ isomorphically to the twisted $K$-theory of $T^{\wedge} \times(G / T)$, where $T^{\wedge}$ is the dual torus to $T$. The twisted $K$-theory is quite easily calculated, and seen to be torsion-free, using the freeness of $R(T)$ as a module over $R(G)$, which I have already mentioned. In fact Michael's papers TwK1 and TwK2] on twisted $K$-theory, written in 2003, were to be his last works in algebraic topology.

The calculations in Tucs] provide abundant support for one of Michael's firmest convictions. When he invented $K$-theory, he could equally well have chosen to fix on what is now called connective $K$-theory $k^{*}$, which has the property that $k^{i}(X)=K^{i}(X)$ when $i \leq 0$, while $k^{i}$ (point) $=0$ for $i>0$. He always asserted that only the periodic theory is 'geometrical', and certainly none of the calculations above have simple analogues in connective $K$-theory.

\section{The CONCEPT OF A $K$-THEORY CLASS}

After the foundational paper [Tucs Michael's next work with Hirzebruch was the pair of papers AnalEmb and AnalCyc concerned with the relation of their new topological $K$-theory to Grothendieck's original $K$-groups for algebraic varieties.

In fact Grothendieck had associated two groups to a variety $X$. The first was the Grothendieck group $K_{\mathrm{coh}}(X)$ of the abelian category of coherent sheaves on $X$, and the second was the Grothendieck group $K_{\mathrm{vb}}(X)$ of the subcategory of vector bundles, i.e., locally free coherent sheaves. The two groups are isomorphic if $X$ is projective and smooth (i.e., nonsingular), for then any coherent sheaf $F$ has a finite resolution - an exact sequence

$$
0 \rightarrow E_{m} \rightarrow \cdots \rightarrow E_{1} \rightarrow E_{0} \rightarrow F \rightarrow 0
$$


in which $E_{0}, E_{1}, \ldots, E_{m}$ are vector bundles - and this expresses $[F]$ as $\sum_{i}(-1)^{i}\left[E_{i}\right]$ in $K_{\mathrm{vb}}(X)$.

Vector bundles can be pulled back by maps, so $K_{\mathrm{vb}}(X)$ is contravariant in $X$, while a coherent sheaf can be pushed forward by a map $f$ (and remains coherent if $f$ is proper). Though it is not said explicitly in [BorS], the analogy of the two Grothendieck groups with homology and cohomology, and, when $X$ is smooth, the interpretation of the resolution of coherent sheaves by vector bundles as Poincaré duality, must surely have been in Grothendieck's mind. The analogy is strengthened because $K_{\mathrm{vb}}(X)$ is a commutative ring under the tensor product, and $K_{\mathrm{coh}}(X)$ is a module over $K_{\mathrm{vb}}(X)$, but is not itself a ring (unless $X$ is smooth) because tensoring coherent sheaves with a vector bundle is an exact functor, but tensoring with a coherent sheaf is not.

Considerations of this kind became much clearer in the language of generalized cohomology theories. Though he invented the name, Michael wrote little about generalized theories beyond recognizing bordism and cobordism in BordCob as a third kind of generalized cohomology (after classical cohomology and $K$-theory). But he was well aware that each theory comes in a homological as well as a cohomological version. He noticed that cobordism arises naturally only in its homological version while $K$-theory is naturally cohomological; much of his subsequent work can be understood as attempts to understand $K$-homology and the Gysin map in $K$-cohomology.

In algebraic geometry the archetypal coherent sheaf on a variety $X$ is the sheaf $\mathcal{O}_{Z}$ of regular functions on an algebraic cycle $Z$ in $X$. Indeed Serre's interpretation of the intersection multiplicity $\left\langle[Z],\left[Z^{\prime}\right]\right\rangle$ of two cycles $Z, Z^{\prime}$ in terms of the derived tensor product of sheaves by his formula

$$
\left\langle[Z],\left[Z^{\prime}\right]\right\rangle=\sum_{i}(-1)^{i} \operatorname{dim}\left(\operatorname{Tor}_{i}^{\mathcal{O}_{X}}\left(\mathcal{O}_{Z}, \mathcal{O}_{Z^{\prime}}\right)\right)
$$

was one of the reasons for the centrality of coherent sheaves in algebraic geometry. The topological substitute for the resolution (2) which associates to $\mathcal{O}_{Z}$-or to any other coherent sheaf supported on $Z$ - an element of the Grothendieck group $K_{\mathrm{vb}}(X)$ is the Thom isomorphism, as Atiyah and Hirzebruch quickly came to realize.

If $Z$ is a closed submanifold of complex codimension $m$ in a compact complex manifold $X$, then the class corresponding to the cycle $[Z]$ in the classical cohomology $H^{2 m}(X)$ is the image of $1 \in H^{0}(X)$ under the composition

$$
H^{*}(Z) \rightarrow H^{*+2 m}(N, N-Z) \cong H^{*+2 m}(X, X-Z) \rightarrow H^{*+2 m}(X),
$$

where $N$ is the normal bundle of $Z$ in $X$, and in the middle we have an excision isomorphism. The left-hand map is the Thom isomorphism-notice that, by excision and homotopy invariance, $H^{*}(N, N-Z)$ is the same as $H_{\mathrm{cpt}}^{*}(N)=H^{*}\left(Z^{N}, \infty\right)$.

The papers AnalEmb and AnalCyc study the corresponding sequence in $K$ theory. The key tool is the understanding of a relative class in $K^{0}(X, Y)$ as a difference-bundle

$$
(E, F ; \alpha: E|Y \cong F| Y) \in K^{0}(X, Y),
$$

where $E$ and $F$ are vector bundles on $X$ identified by $\alpha$ on the subspace $Y$ of $X$. There are several variants of this idea. Because of the flexibility of continuous sections, it is easy to see that the bundle-homomorphism $\alpha$ can be assumed to be defined over all of $X$, while being required to be an isomorphism only over $Y$. A 
further step shows that an element of $K^{0}(X, Y)$ can be defined by a complex of vector bundles

$$
0 \longrightarrow E_{0} \stackrel{d_{0}}{\longrightarrow} E_{1} \stackrel{d_{1}}{\longrightarrow} \cdots \stackrel{d_{m-1}}{\longrightarrow} E_{m} \longrightarrow 0
$$

on $X$ which is exact when restricted to $Y$. Conversely, by introducing fibrewise inner-products on the bundles, it is also easy to see that the $K$-theory element defined by the complex (3) can equally be defined by the difference-construction

$$
\bigoplus_{k} E_{2 k} \stackrel{\oplus\left(d_{2 k}+d_{2 k+1}^{*}\right)}{\longrightarrow} \bigoplus_{k} E_{2 k+1} \text {. }
$$

In algebraic geometry the map $K(Z) \rightarrow K(N, N-Z)$, where $N$ is a complex vector bundle on $Z$, can be defined by the Koszul resolution of a vector bundle $E$ on $Z$ :

$$
\cdots \rightarrow \bigwedge^{3}\left(\hat{N}^{*}\right) \otimes E \rightarrow \bigwedge^{2}\left(\hat{N}^{*}\right) \otimes \hat{E} \rightarrow \hat{N}^{*} \otimes \hat{E} \rightarrow \hat{E} \rightarrow E .
$$

Here $\hat{E}$ and $\hat{N}$ denote the bundles $E$ and $N$ pulled back to the space $N$, and $E$ is regarded as a coherent sheaf on $N$ supported on the zero-section $Z$. The maps in the sequence are given, at a point $\xi \in N$, by the inner-product operation $i_{\xi}: \bigwedge^{i}\left(\hat{N}^{*}\right) \rightarrow \bigwedge^{i-1}\left(\hat{N}^{*}\right)$ on the exterior algebra of the dual of $\hat{N}$, and the sequence (with $E$ omitted) is exact when $\xi$ does not belong to the zero-section $Z$ in $N$. This construction can be transferred directly from algebraic geometry to the topological context. When $E$ is the trivial one-dimensional bundle, it gives a natural Thom class $\theta_{N}^{K, \mathbb{C}}$ for any complex vector bundle $N \rightarrow Z$, and the ratio of $\operatorname{ch} \theta_{N}^{K, \mathbb{C}}$ to the Thom class in classical cohomology is the Todd class of the relative tangent bundle $-N=T_{Z}-\left(T_{X}\right) \mid Z$ of the map $Z \rightarrow X$.

In the paper AnalEmb this procedure is carefully carried out. Because the idea of "excision" is not so simple in algebraic geometry, and because one must be more careful relating the neighbourhood of a submanifold to its normal bundle, it is necessary to invoke deep theorems about the relation between sheaves of algebraic, holomorphic, real-analytic, and smooth functions on a complex variety. The final result is a generalization of the Grothendieck Riemann-Roch theorem to embeddings of compact complex manifolds. The form of the statement is noteworthy. It states that two diagrams commute. The first is the diagram

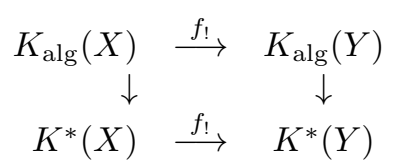

relating the pushforward of coherent sheaves to the Gysin map in $K$-theory-this is the first appearance of the formulation

$$
\text { analytic index }=\text { topological index }
$$

which was to become a hallmark of Michael's work. The second diagram is the purely topological one relating the Gysin maps in $K$-theory and in rational cohomology via the Chern character multiplied by the Todd class. This is the original differentiable Riemann-Roch theorem, which he now saw as a less-interesting computational appendage.

The companion paper AnalCyc treats the same situation, but allows the algebraic cycle $Z$ to be singular. On the smooth projective variety $X$ the structural sheaf $\mathcal{O}_{Z}$ of the cycle is still coherent, and so has a finite resolution by algebraic 
vector bundles on $X$. These bundles can be transferred directly to topological $K$-theory, and so a class $[Z]_{K} \in K^{0}(X)$ is associated to the cycle $Z$. (One must check, of course that it is independent of the chosen resolution.) Michael regarded this as a very interesting result, not only the fact that a singular cycle represents a $K$-theory class, but also because the $K$-theory class $[Z]_{K}$ is a refinement of the classical cohomology class $[Z]$ of the cycle, which is only the leading term of the Chern character of $[Z]_{K}$. If we think of $[Z]$ as a class in the $E_{2}$-term of the AtiyahHirzebruch spectral sequence, it must be annihilated by every differential. Hodge had conjectured that a $2 p$-dimensional cohomology class of an algebraic variety can be represented by an algebraic cycle if it is of type $(p, p)$ when represented by a complex differential form, but the theorem of AnalCyc showed that this could not be true for an integral class which did not survive in the spectral sequence. The paper gave an explicit example of such a class - it was the first (and perhaps the only) occasion when Michael made concrete use of a differential in the spectral sequence. The conclusion was that Hodge's conjecture can at best be true rationally.

The ideas coming from the difference-bundle construction, which had proved so clarifying for the Thom isomorphism, led in other directions too. A $K$-theory class on a compact space $X$ is represented by a virtual vector bundle $E_{0}-E_{1}$. Because of the existence of complementary bundles, it can even be represented by a stable bundle: a stable bundle is an equivalence class of pairs $(E, m)$, where $E$ is a bundle and $m \in \mathbb{Z}$, and $(E, m)$ is equivalent to $\left(E \oplus\left(X \times \mathbb{C}^{k}\right), m+k\right)$. Just as the space of all $n$-dimensional vector spaces has the homotopy type of $B U_{n}$, in the sense that an $n$-dimensional vector bundle on a space $X$ can be pulled back from a universal bundle on $B U_{n}$, so the space of all stable vector spaces is $\mathbb{Z} \times B U$. But stable vector spaces do not have a good notion of direct sum: the sum is defined only up to noncanonical isomorphism. This is reflected in the fact that (though it can be done) it is not completely obvious how to define a 'direct sum' composition-law on $\mathbb{Z} \times B U$ which is coherently homotopy-commutative 7 Representing a $K$-theory class by a map $E_{0} \rightarrow E_{1}$, or by a complex of bundles, even when not thinking of a relative class, brings the advantages of a category with a much better direct sum (and, in the case of complexes, a natural tensor product too).

Fredholm operators. At some point on the road from $K$-theory to index theory, Michael realized that the space of Fredholm operator 8 in a fixed Hilbert space $\mathcal{H}-$ with the norm topology - is an excellent model of the topological category of virtual vector spaces, better than the usual model $\mathbb{Z} \times B U$ of the same homotopy type: a Fredholm operator $F: \mathcal{H} \rightarrow \mathcal{H}$ consists of two finite-dimensional subspaces $\operatorname{ker}(F)$ and $\operatorname{coker}(F)=\operatorname{im}(F)^{\perp}$ of $\mathcal{H}$ together with an isomorphism $\mathcal{H} / \operatorname{ker}(\mathrm{F}) \rightarrow \operatorname{im}(F)$, but the choices of the isomorphism form a contractible space in view of Kuiper's theorem that that the general linear group of Hilbert space is contractible in the norm topology. When we move from a Fredholm operator $F$ to a nearby one $F^{\prime}$, the dimension of the kernel and cokernel may drop, but the change in the virtual vector space, which is their difference, changes continuously, as $F^{\prime}$ induces an isomorphism $\operatorname{ker}(F) / \operatorname{ker}\left(F^{\prime}\right) \rightarrow \operatorname{coker}(F) / \operatorname{coker}\left(F^{\prime}\right)$. This is the starting point of index theory.

\footnotetext{
${ }^{7}$ The need for care here can be seen from the very similar case when " $K$-theory with mod $n$ coefficients" is defined by considering equivalence classes of vector bundles under the relation that two bundles are equivalent if they become isomorphic after adding bundles of the form $E \otimes \mathbb{C}^{n}$. For real vector bundles it turns out unexpectedly that $K O^{0}$ (point; $\left.\mathbb{Z} / 2\right) \cong \mathbb{Z} / 4$.

${ }^{8}$ The result first appeared explicitly in the thesis of Hirzebruch's student Jänich.
} 


\section{BotT PERIODICITY}

Michael saw Bott periodicity as the active ingredient in $K$-theory, and he kept trying to pin down its essence. Bott's original proof had been by Morse theory, and subsequently a very ungeometric proof was given by Moore [M] (cf. also [DL]) by explicitly calculating $H^{*}(B U)$ and $H^{*}(\Omega U)$, and the map between them induced by Bott's map $B U \rightarrow \Omega U$. Michael devised a number of analytic proofs of the theorem, which I shall describe in this section.

A feature of all the analytic proofs is that they carry over without change to equivariant $K$-theory, in the sense that for every finite-dimensional complex representation $V$ of a compact Lie group $G$, we have $K_{G}^{*}(X) \cong K_{G \text {,cpt }}^{*}(X \times V)$. This was crucial in setting up the equivariant theory, where, when $V$ is irreducible, one cannot use induction on the dimension of $V$.

From the first, Michael's preferred statement of Bott's theorem had been as the product formula $K\left(X \times S^{2}\right) \cong K(X) \otimes K\left(S^{2}\right)$, or, equivalently, thinking of $S^{2}$ as the Riemann sphere,

$$
K(X) \cong K(X) \otimes K\left(S^{2}, \infty\right) \cong K\left(X \times S^{2}, X \times \infty\right) .
$$

The group $K\left(S^{2}, \infty\right) \cong \mathbb{Z}$ is generated by the difference element $(L-1)$, where $L$ is the line bundle whose first Chern class generates $\pi_{2}\left(B U_{1}\right)$, and 1 is the trivial line bundle. The Bott map

$$
\beta: K(X) \rightarrow K\left(X \times S^{2}, X \times \infty\right) \subset K\left(X \times S^{2}\right)
$$

is the Gysin map $i$ ! for the inclusion $i: X=X \times 0 \subset X \times S^{2}$, which is given by $i_{!}(E)=E \otimes(L-1)$. After some time, Michael realized with surprise that to prove the theorem it is enough to define a Gysin map

$$
p_{!}: K^{*}\left(X \times S^{2}\right) \rightarrow K^{*}(X)
$$

which is functorial in $X$ and such that $p_{!} \circ i_{!}$is the identity. The reason for this is that $p_{\text {! }}$ can be interpreted as a transformation of cohomology theories $K^{q-2}(X) \rightarrow$ $K^{q}(X)$, while the Bott map $i_{!}: K^{q}(X) \rightarrow K^{q-2}(X)$ is multiplication by an element of $K^{*}$ (point). In this formulation it is clear that $i_{\text {! }}$ and and $p_{\text {! commute, so that }}$ $i_{!} \circ p_{!}=$id follows from $p_{!} \circ i_{!}=\mathrm{id}$.

To define the Gysin map $p_{\text {! }}$ it is natural to begin from the algebro-geometric situation. If $E$ is a bundle on $X \times S^{2}$ whose restriction $E_{x}$ to each subspace $S_{x}^{2}=p^{-1}(x)$ happens to be holomorphic, then we would expect $p_{!}(E)$ to be the virtual bundle whose fibre at $x$ is the virtual vector space $H^{0}\left(S_{x}^{2} ; E_{x}\right)-H^{1}\left(S_{x}^{2} ; E_{x}\right)$. But the dimensions of the two cohomology groups may jump as $x$ changes - even if $X$ is a complex manifold and $E$ is globally holomorphic-and so it is clearly better to think of the family of $\bar{\partial}$-operators $\Omega^{0}\left(S_{x}^{2} ; E_{x}\right) \rightarrow \Omega^{0,1}\left(S_{x}^{2} ; E_{x}\right)$, which, in the holomorphic case at least, is a smooth family of Fredholm operators. At this point, however, we can pass directly to the topological situation, for the set of isomorphism classes of bundles $E$ on $X \times S^{2}$ does not change if the bundles are required to have a smooth structure on each $S_{x}^{2}$ (as can be seen by comparing the spaces of classifying maps of such bundles), and for a bundle which is smooth on each fibre, all we need to define a fibrewise $\bar{\partial}$-operator is the choice - another choice from a contractible space - of a smooth connection along each fibre. This is all explained very carefully in the paper PerIndex.

Michael had a hand in inventing at least two other proofs of Bott's theorem. The idea of the earliest, joint with Bott ABPer, was to categorify the winding 
number of a loop $f$ in the unitary group $U_{n}$ (i.e., its class in $\pi_{1}\left(U_{n}\right) \cong \mathbb{Z}$ ) as a vector space, thereby defining a map $\Omega U_{n} \rightarrow \mathbb{Z} \times B U$. If the loop $f$ is parametrized by the complex numbers $z$ of modulus 1 , we can approximate it by a finite Laurent series $f(z)=\sum a_{k} z^{k}$ whose coefficients $a_{k}$ are $n \times n$ matrices. Multiplying $f$ by a power of $z$ simply moves us from one component of $\Omega B U_{n}$ to another, so we may as well assume $f$ is actually a polynomial. If $f$ were scalar-valued, i.e., if $n=1$, the winding number would be the number of roots of $f$ inside the unit circle, i.e., the sum of the residues of $f^{\prime} / f$. In general, $f$ defines a multiplication operator on the space $\mathcal{H}_{+}$of holomorphic $\mathbb{C}^{n}$-valued functions on the closed unit disc in $\mathbb{C}$, and the winding number is the dimension of the quotient space $E_{f}=\mathcal{H}_{+} / f \mathcal{H}_{+}$. This vector space is a module over the polynomial ring $\mathbb{C}[z]$, and it has a primary decomposition $E_{f}=\bigoplus_{\zeta} E_{f, \zeta}$ into pieces corresponding to the roots $\zeta$ of $f$ inside the disc. The dimension of $E_{f, \zeta}$ is the multiplicity of the root $\zeta$, i.e., the residue of $f^{\prime} / f$ at $\zeta$. We think of $E_{f, \zeta}$ as the categorification of the multiplicity. As $f$ varies, the spaces $E_{f}$ form a vector bundle on the space $\Omega U_{n}$, defining a map

$$
\Omega U_{n} \rightarrow \mathbb{Z} \times B U .
$$

This is clearly left-inverse to the Bott map

$$
\operatorname{Gr}\left(\mathbb{C}^{n}\right) \rightarrow \Omega U_{n}
$$

from the Grassmannian of $\mathbb{C}^{n}$, which takes a subspace $V$ of $\mathbb{C}^{n}$ to the loop whose value at $z$ is $z \oplus 1$ wih respect to the decomposition $\mathbb{C}^{n}=V \oplus V^{\perp}$.

In the paper ABPer the main work is devoted to proving that the map $f \mapsto$ $\left\{E_{f}\right\}$ is also right-inverse to the Bott map, but as Michael later realized, this is unnecessary.

The last of Michael's proofs can be thought of as the categorification of spectral flow. It is essentially the argument used in the paper [SkFred] with Singer on skewadjoint Fredholm operators, to which I shall return in $\S 8$. The idea is extremely simple. If $M_{2 n}$ denotes the contractible space of $2 n \times 2 n$ Hermitian matrices $A$ with $\|A\| \leq 1$ (i.e., the eigenvalues of $A$ are in the interval $[-1,1]$ ), let us consider the map

$$
\mathcal{E}_{2 n}: M_{2 n} \rightarrow U_{2 n}
$$

which takes $A$ to $-\exp (\mathrm{i} \pi A)$. This is surjective, but not a bijection, because when $u$ has the eigenvalue 1 , there is an ambiguity in choosing the logarithm $\pm \mathrm{i} \pi$ of -1 . In fact the inverse image of a unitary matrix $u$ is the Grassmannian $\operatorname{Gr}(\operatorname{ker}(u-1))$. The map $\mathcal{E}_{2 n}$ is not a fibration, though if we stratify $U_{2 n}$ by the dimension of $\operatorname{ker}(u-1)$, then $\mathcal{E}_{2 n}$ is a fibration over each stratum. If we now embed $M_{2 n}$ in $M_{2 n+2}$ by $A \mapsto 1 \oplus A \oplus-1$, and $U_{2 n}$ in $U_{2 n+2}$ by $u \mapsto 1 \oplus u \oplus 1$, and take the union as $n \rightarrow \infty$, then we get a map $\mathcal{E}: M \rightarrow U$ all of whose fibres are isomorphic to $\bigcup \operatorname{Gr}\left(\mathbb{C}^{2 n}\right)$, which is a natural model of $\mathbb{Z} \times B U$. The map $\mathcal{E}$ is not a fibration, but it is a quasifibration, and that is enough to show that the fibre is the loop space of the base.

To explain why I have called this the spectral flow argument, let us consider the space of self-adjoint Fredholm operators $A$ in a fixed complex Hilbert space $\mathcal{H}$. We give this space the operator-norm topology. The spectral theorem gives us a canonical decomposition of $A$ as $A_{+}+A_{-}$, where $A_{+}$is a positive operator and $A_{-}$is negative, and the space of these operators has three connected components, according as either or both of $A_{ \pm}$has infinite rank. Let $\mathcal{F}$ denote the component where both have infinite rank. By multiplying $A$ by a positive number, we can 
ensure that $A_{ \pm}$each have at most a finite number of eigenvalues in the open interval $(-1,1)$, and we shall tacitly restrict to the subspace of such operators, which does not change the homotopy type of $\mathcal{F}$. Thus we assume that any $A \in \mathcal{F}$ has a canonical decomposition

$$
A=A_{+}+A_{0}+A_{-},
$$

where the components have spectra in $[1, \infty),(-1,1),(-\infty,-1]$, respectively.

After all these preliminaries we have the easy

Theorem. The map $A \mapsto \mathrm{e}^{\mathrm{i} \pi A_{0}}$ is a homotopy equivalence $\mathcal{F} \rightarrow \hat{U}$, where $\hat{U}$ is the space of unitary operators $u$ in $\mathcal{H}$ such that $u-1$ has finite rank.

It is easy to see that $\hat{U}$ has the homotopy type of the usual stabilized unitary group that we have denoted $U$. The theorem is proved by showing that the map $\mathcal{F} \rightarrow \hat{U}$ is a quasifibration with contractible fibres: a point of the fibre consists of a decomposition of $(\operatorname{im}(u-1))^{\perp}$ as the orthogonal sum of two infinite-dimensional subspaces together with a positive-definite operator in each.

The fundamental group of $U$ is $\mathbb{Z}$, detected by the winding-number of the determinant of a loop $u_{t}$, or equivalently by the number of times (counted with signs) an eigenvalue of $u_{t}$ crosses a chosen point on the unit circle. This can be called the spectral flow of the loop $u_{t}$, and, when we have a loop of self-adjoint Fredholm operators, the theorem identifies it with the flow through the origin of the eigenvalues of the operator.

An equivalent incarnation of the homotopy-fibration

$$
\mathbb{Z} \times B U \rightarrow\{\text { point }\} \rightarrow U
$$

often arises in quantum field theory (cf. [PS, Chap. 6]). A self-adjoint operator $A \in$ $\mathcal{F}$ defines a polarization of $\mathcal{H}$, i.e., an equivalence class of orthogonal decompositions $\mathcal{H}=\mathcal{H}_{+} \oplus \mathcal{H}_{-}$, and hence a restricted general linear group $G L_{\text {res }}(\mathcal{H})$ consisting of those $g \in G L(\mathcal{H})$ which preserve the polarization. Now $G L_{\mathrm{res}}(\mathcal{H})$ is homotopy equivalent to the space of Fredholm operators in $\mathcal{H}_{+}$, and hence to $\mathbb{Z} \times B U$, by associating to $g$ its $\mathcal{H}_{+} \rightarrow \mathcal{H}_{+}$component. In fact $\mathcal{F}$ is homotopy equivalent to the space of polarizations of $\mathcal{H}$. Thus we have a homotopy-fibration

$$
G L_{\mathrm{res}}(\mathcal{H}) \rightarrow G L(\mathcal{H}) \rightarrow \mathcal{F}
$$

\section{K-HOMOLOGY}

Michael was well aware that for any multiplicative generalized cohomology theory $h^{*}$, the homology $h_{*}(X)$ can be defined as the group of natural transformations $h^{*}(X \times Y) \rightarrow h^{*}(Y)$ of functors of $Y$, and he saw his construction of the Gysin map for a projection $X \times Y \rightarrow Y$ as the definition of an element of $K_{*}(X)$. But of course that 'definition' is not directly illuminating. For a compact space $X$ which can be embedded in $\mathbb{R}^{n}$ as a deformation-retract of an open neighbourhood $U_{X} \subset \mathbb{R}^{n}$ he also knew the definition $h_{*}(X)=h_{\mathrm{cpt}}^{n-*}\left(U_{X}\right)$, which becomes $h_{\mathrm{cpt}}^{n-*}\left(N_{X}\right)$ when $X$ is a smooth manifold with normal bundle $N_{X}$. He did not find that a satisfactory definition either, although, for a manifold $X$ with tangent bundle $T_{X}$, because the sum of two copies of any real vector bundle has a complex structure and hence a natural $K$-theory Thom class, he liked to identify the $K$-theory of the Thom spaces $X^{-T_{X}}$ and $X^{T_{X}}$, and so he did like to think of $K_{*}(X)$ as $K_{\mathrm{cpt}}^{*}\left(T_{X}\right)$, or better, identifying $T_{X}$ with the cotangent bundle $T_{X}^{*}$ by means of a Riemannian metric, 
as $K_{\mathrm{cpt}}^{*}\left(T_{X}^{*}\right)$, which he was soon to regard as the home of the symbols of elliptic pseudodifferential operators on $X$.

Recognizing elliptic differential operators on $X$ as natural elements of $K_{0}(X)$, in the case of a smooth manifold, led Michael to his most serious attempt [EllX] to define $K_{0}(X)$ for a general compact space $X$. He modelled an elliptic pseudodifferential operator on $X$ by a triple $\left(H_{0}, H_{1} ; D\right)$, where $H_{0}$ and $H_{1}$ are Hilbert spaces equipped with the additional structure of being modules over the Banach algebra $C(X)$ of continuous complex-valued functions on $X$, and $D: H_{0} \rightarrow H_{1}$ is a Fredholm operator which is required to commute with multiplication by a function $f \in C(X)$ up to a compact operator (i.e., the commutator $[f, D]$ is compact). The motivation is that for an actual pseudodifferential operator $D$ acting as a Fredholm operator between Sobolev spaces $H_{0}, H_{1}$ of sections of vector bundles on a smooth manifold, the commutator $[f, D]$ with a smooth function $f$ is a pseudodifferential operator of lower order than $D$, and so is compact as an operator $H_{0} \rightarrow H_{1}$. Michael denoted the semigroup of isomorphism classes of triples under direct sum by $\operatorname{Ell}(X)$. It is a covariant functor on the category of compact spaces and continuous maps, and a triple $\left(H_{0}, H_{1} ; D\right)$ is easily seen to define an element of $K_{0}(X)$. Indeed it is not much harder to see that $\operatorname{Ell}(X) \rightarrow K_{0}(X)$ is surjective.

Michael did not develop the theory of $\operatorname{Ell}(X)$ very far: he did not even conjecture what equivalence relation should be put on the triples to get $K$-homology. I suspect that one reason for this was that he could not see how to use $\operatorname{Ell}(X)$ in the way he at first hoped, which was to find a new approach to recent discoveries of Sullivan in geometric topology. It is worth digressing to give a brief account of this.

In 1970 the study of the topology of manifolds was focussed on the relations between smooth, piecewise-linear, and topological structures. In 1956 Thom had shown how to construct rational Pontrjagin classes for a piecewise-linear manifold, beginning from the obsevation that in the rational cohomology of a smooth manifold $X$, the total Pontryagin class and the total $L$-class of $X$ determine each other by Hirzebruch's algebraic calculus. For smooth $X$ the $L$-class can be determined by applying Hirzebruch's signature formula in reverse to all submanifolds of $X$ with trivial normal bundle, i.e., to the inverse images of regular values of smooth maps from $X$ to a sphere. Thom saw that, using a piecewise-linear analogue of transversality, the same procedure works in the PL-category. Subsequently, Novikov proved that the total rational Pontryagin class was invariant under general homeomorphisms of the manifold, and went on to make an important conjecture about the rigidity of the signature of a non-simply-connected manifold. Then, towards the end of the 1960s, after the work of Kirby and Siebenmann determined the small difference between topological and piecewise-linear manifolds, Sullivan synthesized and sharpened much of this work into the following $K$-theoretical statement, which immediately attracted Michael's attention.

Although a smooth manifold needs a spin structure to be orientable for the theory $K O^{*}$, nevertheless for any oriented smooth manifold $X$ there is a canonical element $[X]_{\text {sig }} \in K O_{n}(X)$ whose Chern character is the Poincaré dual of the $L$-class of $X$. It is a generator of $K O_{*}(X)$ as a $K O^{*}(X)$-module if we ignore the prime 2 , i.e., it is an orientation of $X$ for the theory $K O^{*}[1 / 2]=K O^{*} \otimes \mathbb{Z}[1 / 2]$. (The reason for this is best seen in terms of index theory: the symbol of the signature operator is obtained from that of the Dirac operator by tensoring with a vector bundle - in fact with another copy of the spin bundle-whose rank is a power of 
2.) By systematically using these signature orientations, we can introduce Gysin maps $f_{\text {sig }}: K O^{*}(X)[1 / 2] \rightarrow K O^{*}(Y)[1 / 2]$ for all maps $f: X \rightarrow Y$ of oriented smooth manifolds, with the property, of course, that when $Y$ is a point $f_{\operatorname{sig}}(1)$ is the signature of $X$.

Sullivan proved that any oriented topological manifold $X$ has a canonical $K O^{*}[1 / 2]$-orientation which is $[X]_{\operatorname{sig}}$ when $X$ is smooth. Furthermore, if $X$ is given simply as a space satisfying Poincaré duality, then the possible topological manifold structures it possesses (providing $\operatorname{dim}(X)>4$ ) correspond precisely to $h^{*}$ orientations of $X$ for a multiplicative cohomology theory $h^{*}$ which coincides with $K O^{*}$ away from the prime 2 . Sullivan's methods were far from index theory, and completely alien to Michael, who immediately began to hope that a good description of $\mathrm{KO}$-homology might make it possible to find a direct geometric definition of $[X]_{\mathrm{sig}}$ for a topological manifold. He did not make progress himself with this idea, but his hope was justified when, a few years later, Sullivan and Teleman ST] carried it through precisely, combining Sullivan's proof that a topological manifold of dimension $\neq 4$ has a unique Lipschitz structure, with Teleman's development of Hodge theory for Lipschitz manifolds (which rested in turn on earlier work of Whitney). Meanwhile, Michael had supervised the thesis of his student Lusztig [L], which was an important contribution to the index-theoretical understanding of the signature class, and gave a quite new proof of the simplest case of Novikov's conjecture.

At this time Michael became very interested in the $K$-theory of $C^{*}$-algebras and von Neumann algebras 9 and in particular in the ideas of Brown, Douglas, and Fillmore $\mathrm{BDF}$. Although Michael did not pursue his definition of $\operatorname{Ell}(X)$, it was the beginning of a great deal of work by others. Because $X$ entered only through the $C^{*}$-algebra $C(X)$, the definition applied to an arbitrary $C^{*}$-algebra just as well as to $C(X)$. Connes $[\mathrm{C}$ built up an extensive theory which assigned cyclic cohomology classes to a version of Michael's Fredholm triples, and they were the starting point of his important spectral triple definition of a noncommutative manifold [CM]. But it was Kasparov $[\mathrm{K}]$ who completed the task of defining $K$-homology along the lines of Michael's suggestion, and went further to develop a bivariant theory defined for pairs of $C^{*}$-algebras, which, for two commutative algebras $C(X)$ and $C(Y)$, reduces to the group of morphisms $Y_{+} \rightarrow X_{+} \wedge \mathbf{K}$ in the stable homotopy category, where $\mathbf{K}$ is the spectrum representing $K$-theory, and $X_{+}$and $Y_{+}$denote $X$ and $Y$ with an additional disjoint basepoint adjoined.

\section{EQUIVARIANT $K$-THEORY}

The idea of equivariant $K$-theory arose out of the relation of $K^{*}(B G)$ to the representation ring $R(G)$ of a compact Lie group $G$. When $G$ acts on a compact space $X$, an equivariant vector bundle $E$ on $X$-one where $G$ acts on the total space $E$ by bundle maps which cover its action on $X$-is a natural interpolation between a vector bundle and a representation of $G$. In the more modern language of stacks, an equivariant bundle is a bundle on the quotient stack of $X$ by $G$, i.e., it is a representation of the topological groupoid $X / / G$ whose space of objects is $X$ and space of morphisms is $G \times X$, with $(g, x)$ being a morphism from $x$ to $g x$. If the action of $G$ is free, then $E / G$ is a vector bundle on $X / G$, and equivariant bundles on $X$ need not be distinguished from bundles on $X / G$, but in general an

\footnotetext{
${ }^{9}$ Cf. Dan Freed's accompanying account in $\mathrm{Fr}$.
} 
equivariant bundle $E$ carries more information, in the form of a representation of the isotropy group $G_{x}$ of $x$ on the fibre $E_{x}$ at each point $x \in X$.

The groupoid $X / / G$ has its 'realization' $|X / / G|$, which is the space $X_{G}=X \times_{G}$ $E G$ fibred over the classifying space $B G$ with fibre $X$. In other language, this is the homotopy quotient of $X$ by the action of $G$; it is also called the Borel construction from its use $\mathrm{Bor}$ in the study of transformation groups. In exactly the way that a representation $V$ of $G$ defines a vector bundle $E_{V}$ on $B G$, an equivariant bundle $E$ on $X$ defines a vector bundle $E_{G}$ on $X_{G}$.

An equivariant generalized cohomology theory $K_{G}^{*}$ can be defined on the category of compact $G$-spaces in exact analogy to the definition of nonequivariant $K$-theory. Apart from an equivariant version of Bott periodicity, already mentioned in $\S 4$ above, the one new fact needed to get started is that an equivariant bundle $E$ on a compact $G$-space $X$ always has a complementary bundle $F$ such that $E \oplus F$ is trivial in the sense that it is a product $X \times V$, where $V$ is a representation of $G$. (Michael said Dixmier had explained to him how this follows from the Peter-Weyl theorem.)

When evaluated on a point, $K_{G}^{*}$ clearly gives the representation ring $R(G)$ in degree 0 , and vanishes in degree 1 . Two other basic properties should be mentioned. First, when $G$ acts freely on the space $X$, the remark above about equivariant bundles gives us $K_{G}^{*}(X) \cong K^{*}(X / G)$. Then, if $H$ is a subgroup of $G$, a similar elementary equivalence between bundles shows that

$$
K_{H}^{*}(X) \cong K_{G}^{*}\left(G \times_{H} X\right) .
$$

An important particular case is the isomorphism $K_{G}^{*}(G / H) \cong R(H)$. If $H$ is the maximal torus $T$ of a connected group $G$, then $G / T$ is a complex manifold, and we can associate a Gysin map $p_{!}: R(T) \rightarrow R(G)$ to the projection $p: G / T \rightarrow$ (point). This is the subject of the Borel-Weil-Bott theorem as set forth in Bott's paper [Bo], which had a great influence on Michael's thinking. The basis elements of $R(T)$ are the lattice $\hat{T}$ of weights of the group $G$. A weight $\lambda \in \hat{T}$ is dominant if it defines a positive line-bundle $L_{\lambda}$ on $G / T$, and then the cohomology $H^{i}\left(G / T ; L_{\lambda}\right)$ vanishes when $i>0$, while the space of holomorphic sections $H^{0}\left(G / T ; L_{\lambda}\right)$ is the irreducible representation $V_{\lambda}$ of $G$ with highest weight $\lambda$-the representation holomorphically induced from the representation $\lambda$ of $T$. We shall see in a moment how Weyl's formula for its character appears naturally from equivariant $K$-theory.

With the aid of equivariant $K$-theory the proof Cpln that $R(G)^{\wedge} \cong K^{*}(B G)$ for a general compact Lie group $G$ melted away into a dévissage that could almost have been a parody of Grothendieck's style. First, because $G$ can be embedded in a unitary group $U_{n}$, and $K_{G}^{*}(X) \cong K_{U_{n}}^{*}\left(U_{n} \times_{G} X\right)$, we find it is enough to prove the result when $G=U_{n}$. But then, if $\mathbb{T}^{n}$ is the maximum torus of $U_{n}$, the map $K_{U_{n}}^{*}(X)^{\wedge} \rightarrow K^{*}\left(X_{U_{n}}\right)$ is obtained from $K_{\mathbb{T}^{n}}^{*}(X)^{\wedge} \rightarrow K^{*}\left(X_{\mathbb{T}^{n}}\right)$ as the part invariant under the action of the $n$th symmetric group $\Sigma_{n}$ on $\mathbb{T}^{n}$. That, in turn, reduces by induction to the case $n=1$, when the theorem is simply the inverse limit (as $m \rightarrow \infty$ ) of the $K_{\mathbb{T}}^{*}$ exact sequence for the pair $\left(D^{2 m}, S^{2 m-1}\right.$ ) consisting of the unit disc in $\mathbb{C}^{m}$ and its boundary sphere, regarded as $\mathbb{T}$-spaces by complex multiplication.

The argument of this proof of the completion theorem was very soon used by Quillen Q2 to prove the Atiyah-Swan conjecture 10 that the Krull dimension of the

\footnotetext{
${ }^{10}$ Made in FiniteG, and independently by Swan.
} 
mod $p$ cohomology ring $H^{*}\left(B G ; \mathbb{F}_{p}\right)$ of a finite group $G$ is the maximum rank of an elementary $p$-subgroup of $G$. (Quillen pointed out to me in wondering admiration that when he told Michael of his proof - at the IAS in 1969-Michael showed nothing but delight.)

More important than the completion theorem, however, is the fact that classes in $K_{G}^{*}(X)$ can be localized at the fixed points of elements or subgroups of $G$. The most obvious statement is that if $Y$ is a closed $G$-invariant subspace of $X$ outside which an element $g$ of $G$ has no fixed points, then the restriction map of $R(G)$-modules $K_{G}^{*}(X) \rightarrow K_{G}^{*}(Y)$ becomes an isomorphism if we invert all the elements of $R(G)$ which do not belong to the ideal $\mathfrak{p}_{g}$ of characters which vanish at $g$. This can be seen from the equivariant analogue of the Atiyah-Hirzebruch spectral sequence which converges to $K_{G}^{*}(X)$, whose $E_{2}$-term is $H^{*}\left(X / G ;\left\{R\left(G_{x}\right)\right\}\right)$. (Here the cohomology has coefficients in a sheaf on $X / G$ whose stalk at the orbit $G x \in X / G$ is the representation ring of the stabilizer $G_{x}$ of $x$, i.e., $K_{G}^{*}\left(G_{x}\right)$.) All the groups in the spectral sequence are $R(G)$-modules, so we can localize it at the ideal $\mathfrak{p}_{g}$. If $g$ is not conjugate to an element of a subgroup $H$, then we can find a character $\chi$ with $\chi(g) \neq 0$ which vanishes on $H$, and so the localization $R\left(G_{x}\right)_{\mathfrak{p}_{g}}$ vanishes if $x$ does not belong to $Y$.

That is not the optimal statement, however. Localization in equivariant $K$ theory is primarily a way of obtaining $K$-theory analogues of the Lefschetz fixedpoint formula of classical cohomology theory. Suppose, for example, that $X$ is a smooth closed $G$-manifold, and we wish to calculate $p_{!}(\xi) \in R(G)$, where $\xi \in$ $K_{G}^{*}(X)$ and $p$ is the map from $X$ to a point. It is enough to calculate the character $\chi$ of $p_{!}(\xi)$ at each $g \in G$. For a chosen $g$, we may as well replace the group $G$ by the closed subgroup $H \subset G$ generated by $g$.

Let $i: Y \rightarrow X$ be the inclusion of the submanifold of fixed points of $g$, and let $N$ be the normal bundle of $Y$ in $X$. Then the endomorphism $i^{*} i_{\text {! }}$ of $K_{H}^{*}(Y)$ is multiplication by the Euler class $e_{N}$ of $N$, which, by definition, is the restriction of the Thom class of $N$ to its zero section $Y$. Thus

$$
e_{N}=\lambda_{-1}(N)=: \sum_{k}(-1)^{i} \wedge^{k}(N) .
$$

The essential observation is that $e_{N}$ becomes invertible when we localize at $g$ (i.e., at the ideal $\mathfrak{p}_{g}$ ) because, when we restrict it to any point $y \in Y$, its character at $g$ is $\prod(1-\zeta)$, where $\zeta$ runs through the eigenvalues of the action of $g$ on the fibre $N_{y}$ of the normal bundle, none of which can be 1 . Working in $K_{H}^{*}(Y)_{\mathfrak{p}_{g}}$, we then have

$$
p_{!}(\xi)=(p \circ i) !\left(i^{*} i_{!}\right)^{-1} i^{*}(\xi)=(p \circ i) !\left(\frac{i^{*}(\xi)}{e_{N}}\right),
$$

which gives a formula for $p_{!}(\xi)$ purely in terms of data on $Y$.

This argument, based on the invertibility of the Euler class of a normal bundle, was to recur very often in Michael's work, often in studying moduli spaces or localizing path integrals, contexts far from its first use in equivariant $K$-theory. But the first striking application was to the Gysin map $p_{!}: R(T)=K_{G}^{*}(G / T) \rightarrow R(G)$ mentioned above, which expresses the holomorphic induction of representations from a maximal torus $T$ of a connected Lie group $G$. If $\xi \in R(T)$, then it is enough to calculate the value of the character of the induced representation $p_{!}(\xi)$ of $G$ at a generic conjugacy class in $G$, and thus at an element $t \in T$ whose powers are dense in $T$. But the fixed manifold $Y$ of $t$ on $G / T$ is the finite set of elements of the Weyl 
group $W=N(T) / T$, where $N(T)$ is the normalizer of $T$. The normal space $N_{w}$ at a point $w \in W$, as a representation of $T$, has the character $t \mapsto(\mathfrak{g} / \mathfrak{t})\left(w^{-1} t w\right)$ in terms of the adjoint representations of the Lie algebras. This gives us the Weyl character formula

$$
p_{!}(\xi)=\sum_{w \in W} w \cdot\left\{\xi / \lambda_{-1}(\mathfrak{g} / \mathfrak{t})\right\} .
$$

Looking at the character formula in this way was probably the first step towards Michael's interest in the representation theory of semisimple Lie groups, which culminated in his work with Schmid on the construction of the discrete series by means of the Dirac operator.

A serious limitation of equivariant $K$-theory is that it applies only to compact groups $G$. It is essentially a hybrid theory, in which the spaces are treated homotopically but the groups are not. It was very well adapted to the kinds of applications Michael at first envisaged. (For some time, for example, he had in his sights the Feit-Thompson theorem that finite groups of odd order are soluble. This can be restated as a fixed-point theorem: a linear action of a group of odd order on a complex projective space always has a fixed point - and the odd order of the group might perhaps have been crucial for the existence of an equivariant spin structure.) But geometric topology is most often concerned with actions of noncompact groups, especially with the action of the fundamental group of a manifold on its universal cover. When Michael's focus changed from algebraic topology to index theory, he and Bott proved a Lefschetz fixed-point theorem for a map which was not required to belong to a compact group of transformations, but I shall leave that and other techniques he developed for going beyond the realm of equivariant $K$-theory to Dan Freed's accompanying account $[\mathrm{Fr}$.

It seems appropriate, nevertheless, to say something about the paper Sig] on the multiplicativity of the signature when a compact oriented manifold $M$ is fibred over another oriented manifold by a map $p: M \rightarrow X$ with fibre $Y$. Let us assume all three manifolds are even dimensional. As was mentioned in $\S 5$, by systematically using the signature orientation, we can associate a Gysin map $f_{\text {sig }}$ in real or complex $K$-theory (with the prime 2 inverted) to any map $f$ of oriented manifolds. If $q: X \rightarrow$ (point), then the signature of $M$ is given by

$$
(q \circ p)_{\mathrm{sig}}(1)=q_{\mathrm{sig}}\left(p_{\mathrm{sig}}(1)\right)
$$

In a sense, the paper $[\mathrm{Sig}]$ is about how this factorization should be interpreted, but that is not made altogether explicit, as the main thrust is the use of classical algebraic geometry to construct examples where $\operatorname{sig}(M) \neq \operatorname{sig}(X)$. $\operatorname{sig}(Y)$. To understand the factorization (44) we must recognize that the cohomology along the fibres of $p: M \rightarrow X$ is a vector bundle on $X$ equipped with a nondegenerate bilinear form - symmetric or skew according as $\operatorname{dim}(Y)$ is divisible by 4 or not. In either case such a bundle defines an element $p_{\text {sig }}(1) \in K(X)$. The bundle is canonically flat, but in general the flat structure is not compatible with any unitary structure. Its Chern character, therefore, cannot be obtained from the curvature in the usual Chern-Weil way, and need not be trivial. When we apply $q_{\text {sig }}$ to $p_{\text {sig }}(1)$, the result is the signature of the bilinear form on the twisted cohomology $H^{*}\left(X ; p_{\mathrm{sig}}(1)\right)$ with coefficients in the flat bundle. This is the index of the signature operator tensored with the bundle $p_{\text {sig }}(1)$, and the nontriviality of $p_{\text {sig }}(1)$ accounts for the difference between $\operatorname{sig}(M)$ and $\operatorname{sig}(X) \operatorname{sig}(Y)$. It can be calculated by the index theorem, though it is not a case of Hirzebruch's original signature theorem. 
The paper Sig] was the starting point for Lusztig's thesis $[\mathrm{L}$, already mentioned in $\S 5$, which clarified the situation considerably. The point which emerges is that the signature of a closed $n$-manifold should not really be regarded as an element of $K O^{-n}$ (point): its natural home is not $K$-theory but $L$-theory, a different cohomology theory constructed from modules with nondegenerate quadratic forms by using the relation of Witt equivalence, which makes hyperbolic forms trivial. An element of $L^{-n}$ (point) can be represented by a cochain complex satisfying Poincaré duality in dimension $n$. The Gysin maps $p_{\text {sig }}$ and $q_{\text {sig }}$ are really those of $L$-theory. When the prime 2 is inverted, the $L$-theory spectrum coincides with the $K O$-theory spectrum, but, analytically, interpreting the signature as an 'index' is not completely natural, because it involves choosing a Hodge $*$-operator depending on a Riemannian structure which cannot be chosen invariantly under diffeomorphisms of the manifold.

We can think of these invariants of non-simply-connected Poincaré spaces $X$ in terms of $\pi$-equivariant theories, where $\pi=\pi_{1}(X)$. It is natural to map the orientation class $[X]_{\operatorname{sig}} \in L_{n}(X)$ into $L_{n}(B \pi)$ by the classifying map $X \rightarrow B \pi$. There is then a further map from $L_{*}(B \pi)$ to the $L$-theory of the group-ring $\mathbb{Z}[\pi]$ : it is called the assembly map and corresponds to the $K$-theory map $K_{*}(B G) \rightarrow R(G)$ which is the homological version of the map $R(G) \rightarrow K(B G)$ for a compact group $G$. But as it is needed here for the discrete, usually noncompact, group $\pi$, it must be treated by methods very different from equivariant $K$-theory. (When $\pi$ is finite, however, the thesis $\mathrm{W}$ of George Wilson, which followed up a suggestion Michael made at this time, showed how equivariant $K$-theory could be successfully applied.)

In the later period of Michael's mathematical life, when he was mainly concerned with the topology of moduli spaces arising in gauge theory, and with the localization of the infinite-dimensional path-integrals of quantum field theory, equivariant cohomology continued to play a prominent role in his work, though then it was mostly classical equivariant cohomology rather than equivariant $K$-theory. A variety of examples are described in Simon Donaldson's account in this volume Do.

Classical equivariant cohomology $H_{G}^{*}(X ; A)$ is usually defined as the ordinary cohomology $H^{*}\left(X_{G} ; A\right)$ of the homotopy-quotient $X_{G}$, though there are other equivalent definitions (e.g., when $G$ is finite, one can use a free resolution of the cochain complex of $X$ over the group ring of $G$, and for a Lie group $G$ there is an equivariant version of the de Rham complex which calculates $H_{G}^{*}(X ; A)$ when $X$ is a manifold and $A=\mathbb{R}$ or $\mathbb{C}$ ). Because it is defined in terms of the classifying space $B G$, classical equivariant cohomology has no need to restrict itself to compact groups.

But, more obviously than in the nonequivariant case, an equivariant vector bundle is a much more 'natural' object than any version of a classical equivariant class. A striking example of this - the subject of the next section - arises in the construction of cohomology operations. A bundle $E$ has its external $n$th power $E^{\bigotimes n}$, which is a bundle on $X^{n}$ equivariant under the $n$th symmetric group, which acts by permuting the factors. All the operations in $K$-theory are easily constructed from these powers, and we shall see that in a sense they contain as much information as the more complicated Steenrod operations in classical cohomology.

\section{Operations In $K$-THEORY}

Michael's systematic interest in operations in $K$-theory began comparatively late. As soon as $K$-theory had been invented, Adams defined the Adams operations 
(though they were known earlier to Grothendieck) and used them brilliantly to solve the problem of the vector fields on spheres Ad3. Subsequently, Michael used the Adams operations to give a one-page $K$-theory solution $\mathrm{Ad}$-At of the Hopf invariant problem, replacing Adams's very long and difficult proof [Ad1, which used secondary operations in classical cohomology. The new idea used little more than that the Adams operations commute with each other. Michael was immensely proud of it, for it vindicated his conviction that $K$-theory was superior to classical cohomology for solving geometric problems. The argument goes as follows.

Given a map $f: S^{4 n-1} \rightarrow S^{2 n}$, we can use it to attach a $4 n$-cell to the sphere $S^{2 n}$ to form a space $X_{f}$ which fits into a cofibration $S^{2 n} \rightarrow X_{f} \rightarrow S^{4 n}$. Its reduced $K$-theory is $\mathbb{Z} \oplus \mathbb{Z}$, generated by $x$ and $y$ corresponding to $S^{2 n}$ and $S^{4 n}$. Then $x^{2}=h_{f} y$, where $h_{f} \in \mathbb{Z}$ is the Hopf invariant of $f$. We want to show that if $n>4$, then $h_{f}$ must be even.

Because the operation $\psi^{k}$ acts on $S^{2 r}$ by multiplication by $k^{r}$, we must have $\psi^{k}(x)=k^{n} x+a_{k} y$ for some integer $a_{k}$, and then

$$
\psi^{m}\left(\psi^{k}(x)\right)=k^{n}\left(m^{n} x+a_{m} y\right)+a_{k} m^{2 n} y,
$$

from which, using $\psi^{k} \psi^{m}=\psi^{m} \psi^{k}$, we obtain

$$
a_{k} m^{n}\left(m^{n}-1\right)=a_{m} k^{n}\left(k^{n}-1\right) .
$$

Taking $m=2$, we find that if $a_{2}$ is odd, then $k^{n} \equiv 1\left(\bmod 2^{n}\right)$ for all odd $k$. This is impossible if $n>4$, for the multiplicative group of residues mod $2^{n}$ contains an element of order $2^{n-2}$, which is greater than $n$. Finally, $a_{2} \equiv h_{f}(\bmod 2)$, because $\psi^{2}(x) \equiv x^{2}(\bmod 2)$, so the proof is complete.

The paper Ad-At] was submitted a year or so before Michael wrote on $K$-theory operations for their own sake. Meanwhile he and Hirzebruch had written one of the most beautiful papers CohOps of their collaboration-little noticed, perhaps because it is in German - about classical cohomology operations in connection with characteristic classes. That work, as we shall see, led him to think about another important work $\mathrm{Ad2}$ of Adams.

The link between characteristic classes and cohomology operations was known even before the 1950s, especially from Wu's work relating the mod 2 Steenrod operations to Stiefel-Whitney classes 11 Hirzebruch had a great fund of knowlege on this subject before he began collaborating with Michael (cf. $[\mathrm{H}]$ ). In particular, he had calculated the denominator $m_{k}$ of the $k$ th Todd class - a number that we shall see plays an important role in the structure of the space $B U$-and, more importantly, he had seen how the integrality properties of the Todd class at each prime $p$ connect with the Frobenius $p$ th power operation. The essential observation is the following. Let us pass to the $p$-adic completion of the rational numbers, and regard the generating series $\operatorname{Todd}(t)=t /\left(1-e^{-t}\right)$ for the Todd classes as an element of $\mathbb{Q}_{p}[[t]]$. Then, if we adjoin to $\mathbb{Q}_{p}$ an element $\rho=p^{1 /(p-1)}$, the series $\operatorname{Todd}(\rho t)$ has all its coefficients in the subring $A=\mathbb{Z}_{p}[\rho]$ of $\mathbb{Q}_{p}[\rho]$. The quotient ring $A / \rho A$ is the finite field $\mathbb{F}_{p}$. So the series $\operatorname{Todd}(\rho t)$ can be reduced modulo $\rho$ to give a series in $\mathbb{F}_{p}[[t]]$, and we find 12

$$
\operatorname{Todd}(\rho t)=t /\left(t-t^{p}+t^{p^{2}}-t^{p^{3}}+\cdots\right) .
$$

\footnotetext{
${ }^{11} \mathrm{Cf}$. MS, where Milnor uses the Steenrod operations to define the Stiefel-Whitney classes.

${ }^{12}$ The Todd series, the $L$-series, and the $\hat{A}$-series all give rise to exactly the same series when reduced in this way modulo an odd prime $p$.
} 
The paper CohOps treats the relations between classical cohomology operations and characteristic classes from a Riemann-Roch perspective. It begins by determining the group $\mathcal{G}_{p}$ of multiplicative automorphisms of classical cohomology with $\bmod p$ coefficients, where $p$ is a prime. The natural examples of such operations are the total Steenrod square $S q=\sum_{i>0} S q^{i}$ when $p=2$ and the total power $\mathcal{P}=\sum_{i \geq 0} \mathcal{P}^{i}$ when $p$ is odd. (The automorphisms are not required to preserve the grading: we have $S q^{i}: H^{*} \rightarrow H^{*+i}$ and $\mathcal{P}^{i}: H^{*} \rightarrow H^{*+i(2 p-2)}$.)

It turns out that elements $\Phi$ of the group $\mathcal{G}_{p}$ are determined by their action on $H^{*}\left(B \mu_{p} ; \mathbb{F}_{p}\right)$, where $\mu_{p}$ is the group of complex $p$ th roots of unity. In fact $\Phi$ is determined by the power series

$$
\Phi(x) \in \mathbb{F}_{p}[[x]] \subset H^{*}\left(B \mu_{p} ; \mathbb{F}_{p}\right),
$$

where $x$ is the generator of $H^{1}\left(B \mu_{2} ; \mathbb{F}_{2}\right)$ if $p=2$, and is the generator of $H^{2}\left(B \mu_{p} ; \mathbb{F}_{p}\right)$ for odd $p$. The operation $S q$ corresponds to the series $x+x^{2}$, and $\mathcal{P}$ corresponds to $x+x^{p}$. Because it is a natural transformation of theories, any operation $\Phi$ must preserve the coproduct - the formal group law - in $\mathbb{F}_{p}[[x]]$ coming from the product on the space $B \mu_{p}$ coming from addition in $\mu_{p}$. This forces the series $\Phi(x)$ to be of the form

$$
x+a_{1} x^{p}+a_{2} x^{p^{2}}+a_{3} x^{p^{3}}+\cdots,
$$

and $\mathcal{G}_{p}$ is the group of all formal power series of this form. Composition in $\mathcal{G}_{p}$ is the substitution of one series in another: the inverse of the operation $\mathcal{P}$, for example, is given by the series

$$
t-t^{p}+t^{p^{2}}-t^{p^{3}}+\cdots
$$

which we met in equation (5). The fact that all series of the form (6) arise as operations is deduced in CohOps from Milnor's description of the $\bmod p$ Steenrod algebra $13 \mathcal{A}$ as a cocommutative Hopf algebra, but the two statements are equivalent: the graded commutative Hopf algebra $\mathcal{A}_{*}$ dual to $\mathcal{A}$ can be defined by the property that

$$
\operatorname{Hom}_{\text {rings }}\left(\mathcal{A}_{*} ; R\right) \cong \mathcal{G}(R)
$$

for any graded commutative $\mathbb{F}_{p}$-algebra $R$, where $\mathcal{G}(R)$ is the group of series of the form (6), with coefficients in $R$, under substitution.

Atiyah and Hirzebruch define the $W u$ class as the multiplicative characteristic class corresponding to the transformation $\mathcal{P}^{-1}$ of cohomology theories (i.e., the inverse of the total Steenrod operation). They show that when $p=2$, its value on the tangent bundle of a manifold is the class defined much earlier by $\mathrm{Wu}$, and they put Hirzebruch's early results $[\underline{\mathrm{H}}]$ in context by showing that the $\mathrm{Wu}$ class coincides with the $\bmod p$ reduction of the Todd class (or equally well of the $L$-class) given by the series $\operatorname{Todd}(\rho t)$ described above.

The most important theorem in CohOps, however, relates the Riemann-Roch result for the Steenrod operations to the differentiable Riemann-Roch theorem. In studying the relation of $K^{*}(X)$ to $H^{*}(X ; \mathbb{Z})$, Atiyah and Hirzebruch focussed on the case when $H^{*}(X ; \mathbb{Z})$ has no torsion. Then the Chern character embeds $K^{*}(X)$ as a lattice in $H^{*}(X ; \mathbb{Q})$, and the task is to compare this lattice with the lattice $H^{*}(X ; \mathbb{Z})$. Oversimplifying rather crudely, one can say that, modulo a prime $p$, one lattice is obtained from the other by shifting it by the total Steenrod operation. (In

\footnotetext{
${ }^{13}$ When $p$ is odd we mean here the subalgebra of the Steenrod algebra generated by the powers $\mathcal{P}^{i}$ : i.e., omitting the Bockstein operations.
} 
string theory this question became topical when D-branes were invented: in a string background $X$ the D-branes have charges in $H^{*}(X ; \mathbb{R})$, and physicists were surprised to discover that the charges were not the classes of submanifolds of $X$ where the branes were located, but rather belonged to the $K$-theory lattice.) In comparing the two Riemann-Roch statements, Atiyah and Hirzebruch had to appeal to the important paper $\operatorname{Ad2} 14$ in which Adams gave the definitive description of the homotopy type of the space $B U$. This short paper influenced Michael's thinking for some time.

Adams describes $B U$ in terms of its Postnikov tower: for any connected space $X$ there is a 'tower'

$$
X=X\langle 1\rangle \leftarrow X\langle 2\rangle \leftarrow X\langle 3\rangle \leftarrow \cdots,
$$

of spaces, functorial in $X$ up to homotopy, such that $\pi_{i}(X\langle n\rangle) \cong \pi_{i}(X)$ for $i \geq n$, but $\pi_{i}(X\langle n\rangle)=0$ for $i<n$.

In the case of $B U$ we have the Bott equivalences $B U\langle 2 m-2\rangle \rightarrow \Omega^{2} B U\langle 2 m\rangle$, and the Postnikov tower amounts to the fibrations

$$
B U\langle 2 m+2\rangle \rightarrow B U\langle 2 m\rangle \rightarrow H_{2 m},
$$

where $H_{2 m}$ is the Eilenberg-Maclane space $K(\mathbb{Z}, 2 m)$.

The tower $\{B U\langle 2 m\rangle\}$ is essentially the spectrum representing connective $K$ theory $15 k^{*}$, in the sense that

$$
k^{2 m}(X)=[X ; B U\langle 2 m\rangle]
$$

when $m \geq 0$. Recall that the Atiyah-Hirzebruch spectral sequence for $K$-theory is associated to the descending filtration $\left\{K_{(2 m)}(X)\right\}$ of $K(X)$ where $K_{(2 m)}(X)$ consists of the elements which vanish on subspaces on dimension $<2 m$, or equivalently of the maps $X \rightarrow B U$ which lift to $B U\langle 2 m\rangle$. In other words, the connective theory $k^{2 m}(X)$ is a 'representable' version of the filtration

$$
K_{(2 m)}(X)=\beta^{m} k^{2 m}(X) \subset k^{0}(X)=K^{0}(X),
$$

where $\beta$ is the Bott element in $k^{-2}$ (point). The fibration (7) gives us a long exact sequence

$$
\cdots \rightarrow H^{i-1}(X ; \mathbb{Z}) \stackrel{\delta}{\rightarrow} k^{i+2}(X) \stackrel{\times \beta}{\rightarrow} k^{i}(X) \stackrel{\varepsilon}{\rightarrow} H^{i}(X ; \mathbb{Z}) \rightarrow \cdots .
$$

Such a so-called exact couple gives us a spectral sequence. All its groups are $\mathbb{Z}[\beta]$ modules, and it is easily seen to become the usual Atiyah-Hirzebruch spectral sequence when the element $\beta$ is inverted. This formulation shows that the differential $d_{2 r+3}$ of the spectral sequence is the not-everywhere-defined map $\varepsilon \circ\left(\times \beta^{-r}\right) \circ \delta$. (The even differentials, of course, vanish.)

Adams determined the precise integrality properties of the Chern character ch $=\sum \operatorname{ch}_{i}$, with $\operatorname{ch}_{i} \in H^{2 i}(B U ; \mathbb{Q})$. Bott periodicity implies that $\operatorname{ch}_{i}$ becomes an integral class when pulled back to $B U\langle 2 i\rangle$, and Adams defined canonical classes $\operatorname{ch}_{i, r} \in H^{2 i+2 r}(B U\langle 2 i\rangle ; \mathbb{Z})$ which are pullbacks of $m_{r} \operatorname{ch}_{i+r}$, where $m_{r}$ is the number mentioned above which Hirzebruch had found to be the denominator of the $r$ th Todd polynomial. Furthermore, when reduced modulo a prime $p$ Adams showed

\footnotetext{
${ }^{14}$ This was written at about the same time as Michael's paper At-Todd-each refers to the other, and acknowledges stimulating conversations.

${ }^{15}$ This is the multiplicative cohomology theory such that $k^{i}(X)=K^{i}(X)$ when $i \leq 0$, while $k^{i}$ (point) $=0$ when $i>0$.
} 
that the classes $\mathrm{ch}_{i, r}$ for fixed $i$ are all determined from those with $r<p-1$ by applying the Steenrod automorphism $\mathcal{P}^{-1}$. The existence of the integral classes $\mathrm{ch}_{i, r}$ with these properties is enough to fix the complete Postnikov structure of $B U$.

The importance of this result, which Adams proved using detailed knowledge of the Steenrod algebra, spurred Michael to develop a simple direct account PowerOps of the operations in $K$-theory, obtaining most of Adams's results in a more conceptual way. He began by showing that all operations - not just the additive ones - in $K$-theory are obtained from the tensor power operations $\hat{P}^{n}: K(X) \rightarrow K_{\Sigma_{n}}\left(X^{n}\right)$ which were mentioned at the end of $\S 6$. (Here $\Sigma_{n}$ is the $n$th symmetric group.)

The total power operation $\hat{P}=\sum \hat{P}^{n}$ is exponential when multiplication is defined in $\prod K_{\Sigma_{n}}\left(X^{n}\right)$ by the transfer maps

$$
K_{\Sigma_{m}}\left(X^{m}\right) \times K_{\Sigma_{n}}\left(X^{n}\right) \rightarrow K_{\Sigma_{m+n}}\left(X^{m+n}\right),
$$

and the same is true when we restrict from $X^{n}$ to its diagonal subspace to obtain

$$
P^{n}: K(X) \rightarrow K_{\Sigma_{n}}(X)=R\left(\Sigma_{n}\right) \otimes K(X) .
$$

(It is a basic principle that division by $n$ ! in the usual exponential series $\sum x^{n} / n$ ! is replaced, when we categorify, by keeping track of the $\Sigma_{n}$-equivariance of the $n$th tensor power.)

Each element of the group $R_{*}\left(\Sigma_{n}\right)$ of additive maps $R\left(\Sigma_{n}\right) \rightarrow \mathbb{Z}$ evidently gives rise to an operation on $K(X)$. Thus the map $\lambda^{n}$ which counts the multiplicity of the sign representation in a representation of $\Sigma_{n}$ gives the operation $\lambda^{n}: K(X) \rightarrow K(X)$ which takes a vector bundle to its $n$th exterior power, and the Adams operation $\psi^{n}$ corresponds to the map $R\left(\Sigma_{n}\right) \rightarrow \mathbb{Z}$ which evaluates a character on an $n$-cycle. Michael uses Weyl's correspondence between the representations of the symmetric and unitary groups to show that all operations in $K$-theory can be obtained in this way. The tensor power $\left(\mathbb{C}^{k}\right)^{\otimes n}$ defines an element of $R\left(\Sigma_{n}\right) \otimes R\left(U_{k}\right)$, and hence a homomorphism

$$
R_{*}\left(\Sigma_{n}\right) \rightarrow R\left(U_{k}\right) \rightarrow K\left(B U_{k}\right)
$$

and by passing to the limit as $k \rightarrow \infty$, Weyl's correspondence can be reformulated as an embedding of $\bigoplus R_{*}\left(\Sigma_{n}\right)$ in the group $K(B U)$ of all operations in $K$-theory. The image is dense with respect to the filtration topology of $K$-theory. In fact $\bigoplus R_{*}\left(\Sigma_{n}\right)$ is a ring, with its multiplication coming from the transpose of the restriction maps $R\left(\Sigma_{m+n}\right) \rightarrow R\left(\Sigma_{m}\right) \otimes R\left(\Sigma_{n}\right)$, and it is a subring of $K(B U)$, where the natural multiplication corresponds to multiplying the values of operations.

Up to this point Michael was roughly following Grothendieck, who when defining his ring $K_{\text {alg }}(X)$ for an algebraic variety $X$ had emphasized what he called its $\lambda$-ring structure, i.e., the ring of natural operations generated by the exterior power maps $\lambda^{i}: K_{\text {alg }}(X) \rightarrow K_{\text {alg }}(X)$. These maps are not additive: Grothendieck expressed their algebraic properties by introducing a formal indeterminate $t$ and the generating function $\lambda_{t}=\sum_{i \geq 0} \lambda^{i} t^{i}$, and he defined a $\lambda$-structure on a commutative ring $A$ as a map

$$
\lambda_{t}: A \rightarrow 1+t A[[t]],
$$

which is a homomorphism from the additive group $A$ to the multiplicative group of formal power series with constant term 1 in the power-series ring $A[[t]]$, and, more than that, it is a ring-homomorphism for an exotic multiplication $*$ defined on the multiplicative group of power-series, uniquely characterized by naturality in $A$ and 
the property

$$
(1+a t) *(1+b t)=1+a b t .
$$

Grothendieck made a number of observations about the structure of $\lambda$-rings which have since become important in the study of algebraic cycles. He considered $\lambda$-rings which, like the $K$-theory of a connected space, are augmented by a dimensionfunction $A \rightarrow \mathbb{Z}$ whose kernel is a nilpotent ideal. For these $\lambda$-rings he defined the $\gamma$-filtration, a canonical descending filtration of $A$ by ideals $A_{(i)}$ such that $A_{(i)} \cdot A_{(j)} \subset A_{(i+j)}$. This is an algebraic substitute for the topologically defined filtration $\left\{K_{(2 i)}\right\}$ of $K(X)$, which we have mentioned several times. For $A=K(X)$ we have $A_{(i)} \subset K_{(2 i)}(X)$, and the two filtrations coincide rationally, and even coincide exactly if $H^{*}(X ; \mathbb{Z})$ is torsion-free.

Grothendieck defined the Adams operations $\psi^{k}: A \rightarrow A$ by the formula

$$
\sum \psi^{k}(\xi) t^{k}=\frac{\mathrm{d}}{\mathrm{d} t}\left\{\log \lambda_{-t}(\xi)\right\}
$$

which shows they are additive. The operations $\psi^{k}$ commute with each other. They preserve the $\gamma$-filtration, and $\psi^{k}$ acts on $A_{(n)} / A_{(n+1)}$ simply by multiplication by $k^{n}$. This means that $A_{\mathbb{Q}}=A \otimes \mathbb{Q}$ is graded by the eigenspaces of the $\psi^{k}$, and we get a purely algebraic Chern character $A \rightarrow \operatorname{gr} A_{\mathbb{Q}}$. In fact if $A$ is an algebra over $\mathbb{Q}$, then a $\lambda$-structure on $A$ is simply a grading by the natural numbers $\mathbb{N}$, while if $A$ is torsion-free as an abelian group, a $\lambda$-structure is the same as an action of the monoid $\mathbb{N}^{\times}$on $A$ by operations $\psi^{k}$ such that for each prime $p$ we have $\psi^{p}(a) \equiv a^{p}$ $\bmod p$. (This is a theorem of Wilkerson Wilk.)

In Michael's treatment too the Adams operations are central and have the preceding properties with respect to the topological filtration. The new viewpoint makes their additivity more conceptual. For although $P^{n}(a+b)=\sum P^{i}(a) P^{n-i}(b)$, the terms with $0<i<n$ drop out when we apply $\psi^{n} \in R_{*}\left(\Sigma_{n}\right)$, for evaluating a character on the cyclic permutation $g=(12 \cdots n)$ annihilates the image of the transfer from $\Sigma_{i} \times \Sigma_{n-i}$ to $\Sigma_{n}$ because $g$ is not conjugate to any element of $\Sigma_{i} \times \Sigma_{n-i}$.

Indeed we obtain the stronger theorem that $\psi^{n}$ defines a transformation of cohomology theories when we invert the integer $n$. For if we apply $P^{n}$ to the element $\xi \otimes \sigma_{1} \in K_{\mathrm{cpt}}(X \times \mathbb{R})$, where the generator $\sigma_{1} \in K_{\mathrm{cpt}}(\mathbb{R})$ can be thought of as the Thom class, we get 16

$$
P^{n}(\xi) \otimes \sigma_{1} \otimes e_{n} \in K_{\mathrm{cpt}}(X \times \mathbb{R}) \otimes R\left(\Sigma_{n}\right),
$$

where $e_{n}=\lambda_{-1}(N) \in R\left(\Sigma_{n}\right)$ is the Euler class of the normal space to the diagonal $\mathbb{R}$ in the $n$-fold product $\mathbb{R}^{n}$. Consequently, if we identify $K^{2 m}(X)$ with $K(X)$ by Bott periodicity, we obtain a transformation of theories

$$
P^{n}: K^{*}(X) \rightarrow K^{*}(X) \otimes R\left(\Sigma_{n}\right)\left[e_{n}^{-1}\right]=K^{*}(X)[1 / n]
$$

by defining $P^{n}$ on $K^{2 m}(X)$ as the operation $\left(e_{n}\right)^{-m} P^{n}$ on $K(X)$. The last equality here comes from the fact that the character of the virtual representation $e_{n}$ vanishes on all classes in $\Sigma_{n}$ except for the cyclic permutation $(12 \cdots n)$, on which its value is $n$ : thus inverting $e_{n}$ not only makes the power operation additive, but also reduces $P^{n}$ to the Adams operation $\psi^{n}$.

Michael investigated how the total operation $\hat{P}^{n}$ interacts with the filtration of $K_{\Sigma_{n}}\left(X^{n}\right)$, or equivalently of $K\left(\left(X^{n}\right)_{\Sigma_{n}}\right)$. He observed that the associated graded

\footnotetext{
${ }^{16}$ The reason is that the external power $\hat{P}^{n}\left(\sigma_{1}\right)$ is the equivariant Thom class in $K_{\Sigma_{n}, \operatorname{cpt}}\left(\mathbb{R}^{n}\right)$, whose restriction to the diagonal $\mathbb{R}$ is $\sigma_{1} \otimes e_{n}$.
} 
group of the latter is the cellular cochain complex of $\left(X^{n}\right)_{\Sigma_{n}}$, and thereby related $\hat{P}^{n}$ to the operation $H^{m}(X) \rightarrow H^{n m}\left(\left(X^{n}\right)_{\Sigma_{n}}\right)$ in classical cohomology from which the Steenrod powers are derived. But his main result was to refine the basic property $\psi^{p}(\xi) \equiv \xi^{p} \bmod p$ in terms of the filtration of $K(X)$-at least in the case of a torsion-free space whose cohomology can be identified with the graded group of its $K$-theory. If $\xi \in K_{(2 r)}(X)$, so that $\xi^{p} \in K_{(2 p r)}(X)$, then we can find elements $\xi_{i} \in K_{(2 r+2 i(p-1))}$ for $0 \leq i \leq r$ such that $\xi_{0}=\xi$ and $\xi_{r}=\xi^{p}$ and

$$
\psi^{p}(\xi)=p^{r} \xi_{0}+p^{r-1} \xi_{1}+\cdots+\xi_{r} .
$$

Furthermore, each $\xi_{i}$ defines an element of $H^{2 r+2 i(p-1)}(X ; \mathbb{Z})$, and when these are reduced modulo $p$, the resulting classes $\bar{\xi}_{i}$ are uniquely determined by $\xi$, and $\bar{\xi}_{i}=$ $\mathcal{P}^{i}(\bar{\xi})$, where $\mathcal{P}^{i}$ is the usual Steenrod operation. Thus - for a torsion-free spacethe Steenrod operations are just the 'components' of the Adams operation $\psi^{p}$.

In this way Michael obtained the essential results of Adams's paper Ad2, except for the restriction to torsion-free spaces. One important outcome of PowerOps was to inspire Quillen's paper Q3 which used power operations in general complexorientable theories to determine the structure of the complex cobordism ring, by a method in which a generalization of (을 was a crucial step.

Michael made another foray into the theory of $\lambda$-rings in the paper At-Tall written with his student David Tall. This was partly a general exposition of $\lambda$ rings and partly a reworking of Adams's series of papers Ad4, whose aim was to determine the J-homomorphism - the map of homotopy groups $\pi_{i}$ induced by the inclusion $J: O_{n} \rightarrow G_{n}$ of the orthogonal group in the group of homotopy equivalences of the sphere $S^{n-1}$, when $n \gg i$. The groups $\pi_{i}\left(O_{n}\right)$ are known for large $n$ by Bott periodicity, while $\pi_{i}\left(G_{n}\right)=\pi_{i+n}\left(S^{n}\right)$ is the $i$ th homotopy group of the sphere spectrum $\mathbb{S}$. The strategy of $\operatorname{Ad} 4$ was to interpret the induced map $B O \rightarrow B G$ (in the limit as $n \rightarrow \infty$ ) as taking vector bundles to the associated spherical fibrations, up to stable fibre-homotopy equivalence. I shall not say much about this subject, but the ultimate result was that the space $B G$-and the cohomology theory it defines - splits as a product $B(\operatorname{im} J) \times B(\operatorname{coker} J)$, where $\operatorname{im} J$ is the fixed-point spectrum of the action of the Adams operations on $B O$. Adams succeeded in proving this only modulo his conjecture that the Adams operations do not change the stable fibre-homotopy type of a vector bundle-more precisely, because $\psi^{k}$ does not exist as a stable operation until $k$ is inverted, that the map $J_{k}: B O \rightarrow B G[1 / k]$ is homotopic to $J_{k} \circ \psi^{k}$.

The work with Tall addressed only a very special particular case of the larger project. They considered the equivalence relation $\sim$ on complex representations $V$ of a finite $p$-group $P$ for which $V \sim V^{\prime}$ if there is a $P$-equivariant map $S(V) \rightarrow S\left(V^{\prime}\right)$ between the unit spheres of degree prime to $p$. This relation defines a quotient group $J(P)$ of the representation ring $R(P)$. But $R(P)$ can be identified with the representation ring of $P$ over the cyclotomic field $F$ got by adjoining the $|P|$-th roots of unity to $\mathbb{Q}$, and the action of the Adams operations coincides with the action on $R(P)$ of the Galois group $\Gamma$ of $F$. The paper At-Tall established by simple algebraic arguments an isomorphism between $J(P)$ and the coinvariants of the action of $\Gamma$ on $R(P)$.

At the time, the Adams conjecture was regarded as a formidably difficult problem, but in 1969 it was proved independently by Quillen and by Sullivan. Both used étale cohomology, though in rather different ways. But both methods depended on interpreting the Adams operations as Galois actions on algebraic varieties. In that 
sense the approach of At-Tall was prescient. But a few years later a much more elementary proof of the Adams conjecture was found by Becker and Gottlieb BG]. A great deal of lastingly important mathematics was created in the quest to prove Adams's conjecture, and it is amusing to speculate how the subject might have evolved if so many experts had not overlooked the following simple argument of BG.

If a bundle $E$ on $X$ is a sum $L_{1} \oplus \cdots \oplus L_{m}$ of line bundles, then $\psi^{k}(E)=$ $L_{1}^{\otimes k} \oplus \cdots \oplus L_{m}^{\otimes k}$, and the conjecture is true for $E$ because there is an obvious map from $E$ to $\psi^{k}(E)$ of degree a power of $k$ on each fibre. But the same is true if $E$ is only locally a sum of line bundles, and this can be ensured by lifting $E$ to the bundle $Y$ on $X$ whose fibre is the space $Z=U_{m} / N_{m}$ of decompositions of $\mathbb{C}^{m}$ as a sum of lines. (Here $N_{m}$ is the normalizer of the maximal torus of $U_{m}$.) So to prove the Adams conjecture, all one needs is a left-inverse to the map $p: Y \rightarrow X$ in the stable-homotopy category. But in any cohomology theory $h^{*}$ - such as stable cohomotopy - there is a Gysin-type map $p_{*}: h^{*}(Y) \rightarrow h^{*}(X)$ such that $p_{*} \circ p^{*}$ is multiplication by the Euler number of the fibre, which in the case of $Z$ is 1 . (If $h^{*}$ is $K$-theory, for example, $p_{*}$ is given for any bundle of manifolds $p: Y \rightarrow X$ by taking the de Rham complex along the fibres.)

Michael was certainly aware of the existence of this map $p_{*}$ and even that it works universally for any cohomology theory. Furthermore, the argument of At-Tall depends on the fact that any representation of $P$ is induced from a one-dimensional representation of a subgroup of $P$, and this is the same as saying that the action of $P$ is contained in $N_{m}$.

\section{Real $K$-Theory and Clifford algebras}

Alongside $K(X)$ there is the analogous ring $K O(X)$ defined using real rather than complex vector bundles. To make it into a cohomology theory, we need the analogue of Bott periodicity for its representing space $\mathbb{Z} \times B O$. For the orthogonal groups the period is 8 rather than 2: using Morse theory, Bott had found that the successive loop spaces of $\mathbb{Z} \times B O$ are

$$
O, O / U, U / S p, \mathbb{Z} \times B S p, S p, S p / U, U / O, \mathbb{Z} \times B O .
$$

Michael's paper $[\mathrm{ABS}$ with Bott and Shapiro explains how this sequence of spaces can be understood systematically in terms of Clifford algebras. For a finite-dimensional real vector space $V$ with an inner product, the Clifford algebra $C(V)$ is the algebra generated by $V$ with the relations $v^{2}=\langle v, v\rangle$. It is an algebra with a mod 2 grading, in which the elements of $V$ have degree 1 . The grading is important to ensure the relation

$$
C(V \oplus W) \cong C(V) \otimes C(W),
$$

where multiplication in the tensor product algebra is defined by

$$
(a \otimes b)\left(a^{\prime} \otimes b^{\prime}\right)=(-1)^{\operatorname{deg}(\mathrm{b}) \operatorname{deg}\left(\mathrm{a}^{\prime}\right)}\left(a a^{\prime} \otimes b b^{\prime}\right) .
$$

If the inner product of $V$ is positive or negative definite, then a graded $C(V)$-module $E$ defines an element $\theta_{E} \in K O_{\text {cpt }}(V)$ by the difference construction $\left(E^{0}, E^{1} ; \gamma_{V}\right)$, where $E^{i}$ means the trivial bundle $V \times E^{i}$ on $V$, and $\gamma_{V}$ denotes the Clifford multiplication $(v, \xi) \mapsto(v, v \xi)$. All elements of $K O_{\text {cpt }}(V)$ can be obtained in this way, though the module $E$ may not be uniquely determined by the $K O$-theory class. Let us write $C_{n}$ for the algebra $C\left(\mathbb{R}^{n}\right)$ when $\mathbb{R}^{n}$ is given the negative of the 
usual inner product, so that $C_{n}$ is generated by anticommuting elements $\gamma_{1}, \ldots, \gamma_{n}$ such that $\gamma_{i}^{2}=-1$. If a graded $C_{n}$-module $E$ admits an extra endomorphism $\gamma_{n+1}$ making it a graded $C_{n+1}$-module, then the difference element $\left(E^{0}, E^{1} ; \gamma_{\mathbb{R}^{n}}\right) \in$ $K O_{\text {cpt }}\left(\mathbb{R}^{n}\right)$ vanishes, for the multiplication map by $v \in \mathbb{R}^{n}$ can be deformed to the everywhere-invertible multiplication by $v+\gamma_{n+1}$. In fact it turns out that we have an exact sequence

$$
\mathcal{M}_{n+1} \rightarrow \mathcal{M}_{n} \rightarrow K O_{\text {cpt }}\left(\mathbb{R}^{n}\right) \rightarrow 0,
$$

where $\mathcal{M}_{n}$ denotes the Grothendieck group of graded $C_{n}$-modules, and the first map is the restriction from $C_{n+1}$ to $C_{n}$.

In $[\mathrm{ABS}$, the aim is to develop a new systematic account of the spaces (9), and the exactness of (10) is proved by using Bott's earlier work. The successive algebras $C_{n}$, for $n \geq 0$, are easily found to be

$$
\mathbb{R}, \mathbb{C}, \mathbb{H}, \mathbb{H} \oplus \mathbb{H}, \mathbb{H}(2), \mathbb{C}(4), \mathbb{R}(8), \mathbb{R}(8) \oplus \mathbb{R}(8), \mathbb{R}(16), \ldots,
$$

where $\mathbb{H}$ denotes the quaternions, and $A(r)$ is the algebra of $r \times r$-matrices over an algebra $A$. Bearing in mind that the category of graded $C_{n}$-modules is equivalent to the category of ungraded $C_{n-1}$-modules, and also that for any algebra $A$ the categories of $A$-modules and of $A(r)$-modules are equivalent, we see that the classifying spaces for the successive categories of graded $C_{n}$-modules are

$$
(\mathbb{Z} \times B O)^{2}, \mathbb{Z} \times B O, \mathbb{Z} \times B U, \mathbb{Z} \times B S p, \ldots,
$$

while the successive fibres of the forgetful maps from each of these spaces to its predecessor are precisely the sequence (9), e.g., $O$ is the fibre of $\mathbb{Z} \times B O \rightarrow$ $(\mathbb{Z} \times B O)^{2}$, while $O / U$ is the fibre of $\mathbb{Z} \times B U \rightarrow \mathbb{Z} \times B O$, etc.

The Clifford algebra viewpoint makes it easy to understand the multiplicative properties of $K O^{*}$ (point). The representations of the graded algebras $C_{1}$ and $C_{2}$ are just sums of copies of their regular representations. We write $\eta$ for the regular representation of $C_{1}$. Because $C_{1} \oplus C_{1}$ admits an action of $C_{2}$, we have $2 \eta=$ $0 \in K O^{-1}$ (point). The regular representation of $C_{2}$ is $\eta^{2}$ and is the generator of $K \mathrm{O}^{-2}$ (point). On the other hand, the $C_{3}$-module $\eta^{3}$ admits an action 17 of $C_{4}$, so $\eta^{3}=0$ in $K^{-3}$ (point). And so on ....

Another advantage of the Clifford viewpoint is that it gives us a very natural definition of a spin structure on a Riemannian manifold $X$ as a spinor bundle $S$ on $X$, i.e., a bundle of graded modules for the bundle of graded Clifford algebras $C\left(T_{X}\right)$ whose fibre $S_{x}$ at $x \in X$ is an irreducible $C\left(T_{x}\right)$-module. The choice of a connection in a spinor bundle $S$ gives one a real Dirac operator on X. The connection, and hence the Dirac operator, is unique up to homotopy.

The treatment of real $K$-theory in ABS still depends on Bott's Morse theory, but not much later Michael thought of a more ingenious approach which provided a self-contained proof of orthogonal periodicity. The new idea came from algebraic geometry, where a real algebraic variety $X_{\mathbb{R}}$ can be regarded as the fixed points of complex conjugation on the complexification of $X_{\mathbb{R}}$, a complex variety $X$ which is "defined over the real numbers". A real vector bundle on $X_{\mathbb{R}}$ is then the same as a complex bundle on $X$ equipped with an antilinear conjugation map covering the complex conjugation in $X$.

\footnotetext{
${ }^{17}$ Equivalently, an ungraded $C_{2}$-module admits an ungraded action of $C_{3}$, because when $\gamma_{1}$ and $\gamma_{2}$ are given, we can define $\gamma_{3}=\gamma_{1} \gamma_{2}$.
} 
This led Michael to define $K \mathbb{R}(X)$, for any compact space $X$ with an involution, as the Grothendieck group of complex vector bundles $E$ on $X$ equipped with an antilinear involution covering that of $X$. If the involution of $X$ is trivial this is simply the usual group $K O(X)$. The great virtue of the new definition is that the analytic proofs of complex Bott periodicity carry over without change to prove

$$
K \mathbb{R}(X) \cong K \mathbb{R}_{\text {cpt }}(X \times \mathbb{C})
$$

when $X$ has an arbitrary involution and $\mathbb{C}$ has its usual complex conjugation. The next step is to define a bigraded group $K \mathbb{R}^{p, q}(X)=K \mathbb{R}_{\text {cpt }}\left(X \times \mathbb{R}^{p, q}\right)$, where $\mathbb{R}^{p, q}$ is the subspace $i \mathbb{R}^{p} \times \mathbb{R}^{q}$ of $\mathbb{C}^{p+q}$ with its induced conjugation. The periodicity result (11) then shows that $K \mathbb{R}^{p, q}(X) \cong K \mathbb{R}^{p+1, q+1}(X)$, so that $K \mathbb{R}^{p, q}(X)$ depends only on $p-q$. It can therefore be defined for all $p, q$, and, crucially, has the exact sequences of a cohomology theory.

The theory $K \mathbb{R}^{*, *}$ appears naturally in many contexts in analysis, especially because the Fourier transform takes a real-valued function $f$ on $\mathbb{R}$ to a function $\hat{f}$ on $i \mathbb{R}$ with the property that $\hat{f}(\bar{z})$ is the complex-conjugate of $\hat{f}(z)$. In particular, the symbol of a real elliptic pseudodifferential operator on a manifold $X$ is an element of $K \mathbb{R}\left(T^{*} X\right)$, where the involution on the cotangent bundle is given by multiplication by \pm 1 on each fibre.

Michael showed how the 8-fold periodicity of $K O$-theory arises in the framework of $K \mathbb{R}$. His ingenious argument has three steps.

(i) If $S_{\mathbb{H}}$ is the 3 -sphere with the antipodal involution, then the theory $X \mapsto$ $K \mathbb{R}^{*, *}\left(X \times S_{\mathbb{H}}\right)$ is 8 -fold periodic in $p-q$. This follows from

$$
K \mathbb{R}^{*, *+4}\left(X \times S_{\mathbb{H}}\right) \cong K \mathbb{R}^{*+4, *}\left(X \times S_{\mathbb{H}}\right),
$$

which holds because quaternionic multiplication - thinking of $S_{\mathbb{H}}$ as the unit quaternions - gives us an isomorphism of spaces with involution

$$
S_{\mathbb{H}} \times \mathbb{R}^{0,4} \rightarrow S_{\mathbb{H}} \times \mathbb{R}^{4,0} .
$$

(ii) There is a natural exact sequence

$$
0 \rightarrow K \mathbb{R}^{*, *}(X) \rightarrow K \mathbb{R}^{*, *}\left(X \times S_{\mathbb{H}}\right) \rightarrow K \mathbb{R}^{*-4, *+1}(X) \rightarrow 0 .
$$

This is part of the exact sequence for the pair of spaces formed by the unit disc and unit sphere in $\mathbb{R}^{4,0}$. The group on the right is the relative group for the pair, i.e.,

$$
K \mathbb{R}_{\mathrm{cpt}}^{*, *+1}\left(X \times \mathbb{R}^{4,0}\right) \cong K \mathbb{R}_{\mathrm{cpt}}^{*, *+5}\left(X \times \mathbb{R}^{4,4}\right) \cong K \mathbb{R}^{*-4, *+1}(X) .
$$

The long exact sequence becomes a short exact sequence because the map $K \mathbb{R}^{*-4, *}(X) \rightarrow K \mathbb{R}^{*, *}(X)$ is multiplication by the restriction to $\mathbb{R}^{0,4}$ of the Thom class in $K \mathbb{R}_{\text {cpt }}\left(\mathbb{R}^{4,4}\right)$. This is just the fourth power of the restriction to $\mathbb{R}^{0,1}$ of the Bott element in $K \mathbb{R}_{\text {cpt }}\left(\mathbb{R}^{1,1}\right)$, i.e., it is $\eta^{4}=0$, where $\eta \in K \mathbb{R}_{\text {cpt }}(\mathbb{R})=\mathbb{Z} / 2$ is the standard generator mentioned above.

(iii) The final step is to prove the commutativity of the diagram

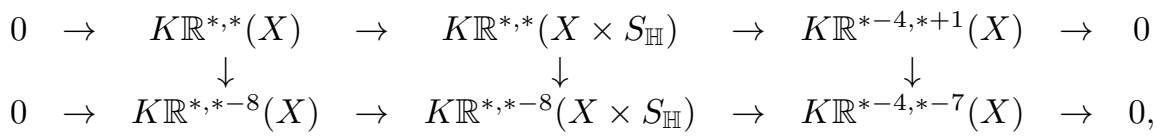

where the outside vertical maps are multiplication by the generator of $K \mathbb{R}^{-8}$ (point) and the vertical map in the middle is the 8-fold periodicity established in the first step of the proof. Given the commutativity, the bijectivity of the outside vertical maps follows from that of the middle map. The commutativity is 
checked by an explicit examination of the Clifford algebra modules, which I shall omit.

It seems a little clumsy that the shifted theory $K O^{*-q}$ should appear as the relative theory of the restriction from the $K$-theory of $C_{q+1}$-modules to that of $C_{q}$-modules. The situation looks much more elegant and natural when we think in terms of Fredholm operators. Recall (from the end of $\S 3$ ) that the space of Fredholm operators in a real or complex Hilbert space is a model for the space of virtual vector spaces and, hence, is a representing space for real or complex $K$ theory. Let us now take a mod 2 graded real Hilbert space $\mathcal{H}_{q}$ which is a graded $C_{q}$-module on which the generators $\gamma_{i}$ act by skew-adjoint operators of degree 1 for definiteness, let us assume $\mathcal{H}_{q}=C_{q} \otimes \mathcal{H}$, where $\mathcal{H}$ is a fixed graded real Hilbert space with infinite-dimensional even and odd components but no $C_{q}$-action.

Let $\mathcal{F}_{q}$ denote the space of skew-adjoint operators of degree 1 in $\mathcal{H}_{q}$ which are maps of $C_{q}$-modules, i.e., which anticommute with each $\gamma_{i}$. Notice that, by the Morita equivalence between $C_{q}$-modules and $C_{q+8}$-modules, the spaces $\mathcal{F}_{q}$ and $\mathcal{F}_{q+8}$ are identical. Atiyah and Singer SkFred proved

Theorem. The space $\mathcal{F}_{q}$ is a representing space for $\mathrm{KO}^{-q}$.

If we accept the result (10), then this theorem is fairly obvious. The essential point is that if $A \in \mathcal{F}_{q}$ is invertible, then $B=-A^{2}$ is a positive-definite self-adjoint operator of degree 0 in $\mathcal{H}_{q}$ which commutes with the $C_{q}$-action, and so we can extend the $C_{q}$-action on $\mathcal{H}_{q}$ to a $C_{q+1}$-action by defining $\gamma_{q+1}=B^{-1 / 2} A$. Just as an ordinary Fredholm operator is, up to homotopy, a pair $\left(E_{0}, E_{1}\right)$ of finitedimensional vector spaces which, when the Fredholm operator moves, can jump to $\left(E_{0} \oplus F, E_{1} \oplus F\right)$, so the elements of $\mathcal{F}_{q}$ model pairs $\left(E_{0}, E_{1}\right)$ of finite-dimensional $C_{q}$-modules which can jump by the addition of a $C_{q+1}$-module $F$.

The natural examples of elements of $\mathcal{F}_{q}$ are Dirac operators with various additional symmetries. For example, $\mathcal{F}_{1}$ is homeomorphic to the space of real skew Fredholm operators (with no grading), and $\mathcal{F}_{7}$ - slightly more complicatedly - to the space of real self-adjoint Fredholm operators. But this is part of index theory, and I shall leave it to Dan Freed's accompanying account $\mathrm{Fr}$.

More relevant here is that Atiyah and Singer proved directly that there is a homotopy equivalence between $\mathcal{F}_{q+1}$ and the loop space of $\mathcal{F}_{q}$ given by the map

$$
A \mapsto\left\{\theta \mapsto \gamma_{q+1} \cos \theta+A \sin \theta\right\}
$$

This gave a completely new proof of Bott periodicity, in both the real and the complex cases. It is essentially the third of the analytic proofs discussed in $\S 4$, described there as the categorification of spectral flow.

\section{About THE AUTHOR}

Graeme Segal became one of Michael Atiyah's graduate students in 1963 and after that (until 1990) was his colleague at St Catherine's College, Oxford. He is now an emeritus fellow of All Souls College in Oxford, and works in algebraic topology and quantum field theory. 


\section{REFERENCES}

\section{Works with Michael Atiyah as an AUthor}

[ABPer] (with R. Bott) On the priodicity theorem for complex vector bundles, Acta Mathematica 112 (1964), 229-247.

[ABS] (with R. Bott and A. Shapiro) Clifford modules, Topology 3 (Suppl. 1)(1964), 3-38.

[Ad-At] (with J. F. Adams) K-theory andthe Hopf invariant, Quart. J. Math. Oxford 17 (1966), 31-38.

[AnalCyc] (with F. Hirzebruch) Analytic cycles on complex manifolds, Topology 1 (1962), 25-45.

[AnalEmb] (with F. Hirzebruch) The Riemann-Roch theorem for analytic embeddings, Topology 1 (1962, 151-166.

[AtCl] Complex analytic connections in fibre bundles, Trans. Amer. Math. Soc. 85 (1957), 181-207.

[At-Tall] (with D. Tall) Group representations, $\lambda$-rings, and the J-homomorphism, Topology 8 (1969), 253-297.

[At-Todd] (with J. A. Todd) On complex Stiefel manifolds, Proc. Camb. Phil. Soc. 56 (1960), 342-353.

[BordCob] Bordism and cobordism, Proc. Camb. Phil. Soc. 57 (1961), 200-208.

[CohOps] (with F. Hirzebruch) Cohomologie-operationen und charakteristische Klassen, Math. Z. 77 (1961), 149-187.

[Cpln] (with G. Segal) Equivariant K-theory and completion, J. Diff. Geom. 3 (1969), 1-18.

[DiffRR] (with F. Hirzebruch) Riemann-Roch theorems for differentiable manifolds, Bull. Amer. Math. Soc. 65 (1959), 276-281.

[EllCve] Vector bundles over an elliptic curve, Proc. London Math. Soc. 7 (1957), 414-452.

[EllX] Global theory of elliptic operators, Proc. Int. Symp. on Functional Analysis, Tokyo, University of Tokyo Press (1969), 21-30.

[FiniteG] Characters and cohomology of finite groups, Publ. Math. I.H.E.S. Paris (1961), 247289.

[ImmEmb] Immersions and embeddings of manifolds, Topology 1 (1962), 125-132.

[KTh] K-theory, Benjamin, New York (1967).

[Min] Roberto Minio, Interview with Michael Atiyah, The Mathematical Intelligencer 6 (1984), 9-19.

[PerIndex] Bott periodicity and the index of elliptic operaators, Quart. J. Math. Oxford 19 (1968), 113-140.

[PowerOps] Power operations in K-theory, Quart. J. Math. Oxford 17 (1966), 165-193.

[SkFred] (with I. M. Singer) Index theory for skew-adjoint Fredholm operators, Publ. Math. I.H.E.S. Paris 37 (1969), 305-326.

[Sig] The signature of fibre bundles, in Global Analysis, papers in honour of K. Kodaira, University of Tokyo Press and Princeton University Press (1969), 73-84.

[ThCpl] Thom complexes, Proc. London Math. Soc. 11 (1961), 291-310.

[Tucs] (with F. Hirzebruch) Vector bundles and homogeneous spaces, Proc. Symp. Pure Math. 3 Amer. Math Soc., 1961.

[TwK1] (with G. Segal) Twisted K-theory, Ukrainian Bull. Math. 1 (2004), 291-332.

[TwK2] (with G. Segal) Twisted K-theory and cohomology in Inspired by S.S. Chern, P.A. Griffiths (ed.), Nankai Tracts in Math. 11, World Scientific, 2007.

\section{OTHER WORKS}

[Ad1] J. F. Adams, On the non-existence of elements of Hopf invariant one, Annals of Math. 72 (1960), 20-104.

[Ad2] J. F. Adams, On Chern characters and the structure of the unitary group, Proc. Cambridge Phil. Soc. 57 (1961), 189-199.

[Ad3] J. F. Adams, Vector fields on spheres, Annals of Math. 75 (1962), 603-632.

[Ad4] J. F. Adams, On the groups $J(X) I-I V$, Topology 2 (1963), 181-195; 3 (1965), 137-171; 3 (1965), 193-222; 5 (1966) 21-71; 7 (1968), 331.

[BarH] D. Baraglia and P. Hekmati, A Fourier-Mukai approach to the K-theory of compact Lie groups, Adv. Math. 269 (2015), 355-345.

[B] M. G. Barratt, Track groups I, II, Proc. London Math. Soc. 5 (1955), 71-106 and 285-329. 
[BG] J. C. Becker and D. H. Gottlieb, The transfer map and fiber bundles, Topology 14 (1975), $1-12$.

[BDF] L. Brown, R. Douglas, and P. Fillmore, Extensions of $C^{*}$-algebras and $K$-homology, Annals of Math. 105 (1977), 265-324.

[Bor] A. Borel, Seminar on transformation groups, Ann. of Math. Studies 46, Princeton Univ. Press 1960.

[BorH] A. Borel and F. Hirzebruch, Characteristic classes and homogeneous spaces I, II, III, Amer. J. Math. 80 (1958), 458-538; 81 (1959), 315-382; 82 (1960), 491-504.

[BorS] A. Borel and J.-P. Serre, Le théorème de Riemann-Roch, Bull. Soc. Math. France 86 (1958), 97-136.

[Bo] R. Bott, Homogeneous vector bundles, Annals of Math. 66 (1957), 203-248.

[BoM] R. Bott and J. Milnor, On the parallelizability of the spheres, Bull. Amer. Math. Soc. 64 (1958), 87-89.

[C] A. Connes, Noncommutative geometry, Academic Press, San Diego, CA, 1994.

[CM] A. Connes and H. Moscovici, The local index formula in noncommutative geometry, Geom. Funct. Anal. 5 (1995), 174-243.

[Do] S. Donaldson, Atiyah's work on holomorphic vector bundles and gauge theories, Bull. Amer. Math. Soc. 58 (2021), no. 4, 567-610.

[DL] E. Dyer and R. Lashof, A topological proof of the Bott periodicity theorems, Ann. Mat. Pura Appl. (4)54 (1961), 231-254.

[Fr] D. Freed, The Atiyah-Singer index theorem, Bull. Amer. Math. Soc. 58 (2021), no. 4, 517-566.

[H] F. Hirzebruch, On Steenrod's reduced powers, the index of inertia, and the Todd genus, Proc. Nat. Acad. Sci. U.S.A. 39 (1953), 951-956.

[Ho] L. Hodgkin, On the K-theory of Lie groups, Topology 6 (1967), 1-36.

[J] I. M. James, Spaces associated with Stiefel manifolds, Proc. London Math. Soc. 9 (1959), $115-140$.

[K] G. G. Kasparov, Operator K-theory and its applications, Proc. Internat. Congress of Mathematicians, Vol. 1, 2 (Warsaw 1983), 987-1000, PWN, Warsaw, 1984.

[L] G. Lusztig, Novikov's higher signatures and families of elliptic operators, J. Diff. Geom. 7 (1972), 229-256.

[MS] J. Milnor and J. Stasheff, Characteristic classes, Ann of Math. Studies 76, Princeton University Press, 1974.

[M] J. C. Moore, Contribution to Séminaire Henri Cartan de l'École Normale Supérieure 1959-60, Paris.

[PS] A. Pressley and G. Segal, Loop groups, Oxford University Press, 1986.

[P] D. Puppe, Homotopiemengen und ihre induzierten Abbildungen I, Math. Z. 69 (1958), 299-344.

[Q1] D. Quillen, The mod 2 cohomology rings of extra-special 2-groups and the spinor groups, Math. Ann. 194 (1971), 197-212.

[Q2] D. Quillen, The spectrum of an equivariant cohomology ring I, Annals of Math. 94 (1971), $549-572$.

[Q3] D. Quillen, Elementary proofs of some results of cobordism theory using Steenrod operations, Adv. in Math. 7 (1971), 29-56.

[S] R. Steinberg, On a theorem of Pittie, Topology 14 (1975), 173-177.

[ST] D. Sullivan and N. Teleman, An analytic proof of Novikov's theorem on rational Pontrjagin classes, I.H.E.S. Publ. Math. 58 (1983), 79-81.

[W] G. Wilson, K-theory invariants for unitary G-bordism, Quart. J. Math. Oxford 24 (1973), 499-526.

[Wilk] C. Wilkerson, Lambda rings, binomial domains, and vector fields over $\mathrm{CP}(\infty)$, Comm. Algebra 10 (1982), 311-328.

All Souls College, Oxford, United Kingdom 